SERVIÇO DE PÓS-GRADUAÇÃO DC ICMC-USP

Data de Depósito: $\quad 18.05 .2004$

Assinatura: Kauretobuyturi

\title{
Aplicação do modelo t-student para análise dos resultados de ensaios de proficiência ${ }^{1}$
}

Flávia Maria Ravagnani Neves

Orientador: Prof. Dr. Dorival Leão Pinto Junior

Dissertação apresentada ao Instituto de Ciências Matemáticas e de Computação - ICMC-USP, como parte dos requisitos para obtenção do título de Mestre em Cièncias de Computação e Matemática Computacional.

\author{
USP - São Carlos \\ Maio/2004
}


A Comissão Julgadora:

Prof. Dr. Dorival Leão Pinto Jr.

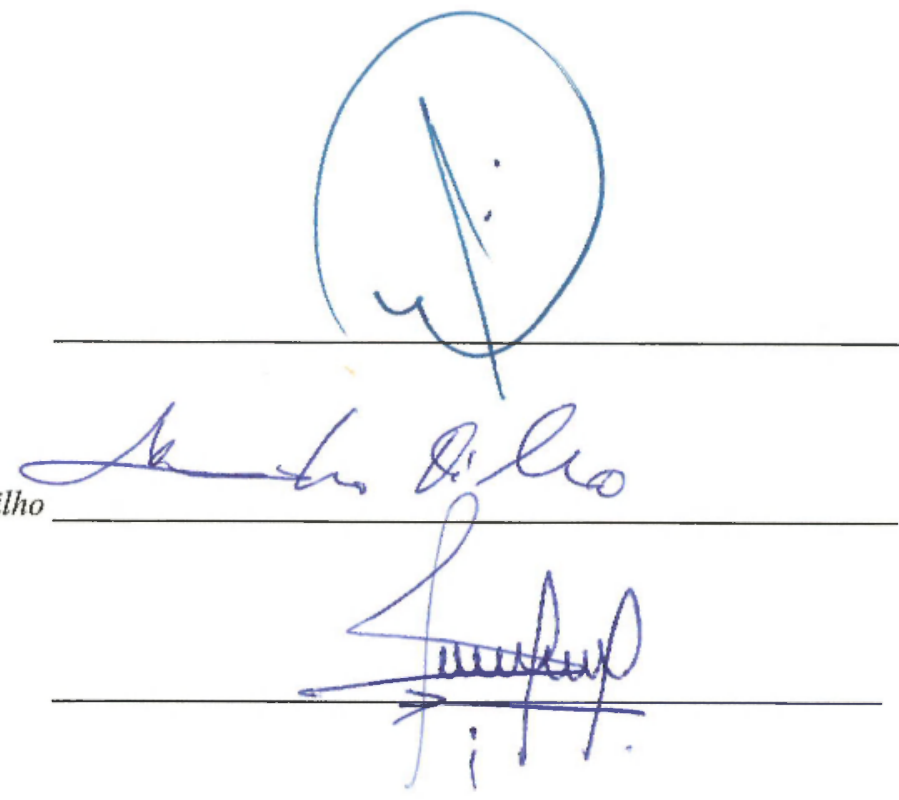


À minha mãe, Luzia Teresa Ravagnani Neves, por ensinar-me a aprender sempre e ao meu pai, João Ferreira Neves, pelo exemplo de dedicação. 


\section{Agradecimentos}

Agradeço a Dens e à Virgem Santíssima, que me acompanharam durante todos estes anos através das orações diárias de minha mãe.

Não posso deixar de destacar o constante apoio c compreensão da minha família. Agradeço, cm especial, aos meus pais João e Luzia, por incentivarem e estimularem meus cstudos e aos mens irmãos, Antônio de Pádua e Cíntia, pela amizade e pelo carinho. Agradeço a todos os mens tios e tias, primos e primas que torceram para que elu conquistasse mais esta etapa.

Agradeço, em particular, ao meu noivo Fabrício. pelo amor e apoio, por confiar no men caráter, na minha competência c: no nosso futuro.

Agradeço ao meu orientador Prof. Dr. Dorival Leão Pinto Junior pela orientação deste trabalho e pelos seus ensinamentos, que foram fundamentais para o término desta dissertação.

Quero manifestar aqui que me sinto muito orgulhosa de ter encontrado durante este período grandes amigos, como o Alex, o Fábio (Patão), a Mariana, o Mário (Ronaldinho), a Grazielle c a Kelly. En especial gostaria de destacar o carinho e a cumplicidade das minhas companheiras de sempre, Aline e Luci.

Agradeço aos mous colegas do mestrado. Gecirlei, Fabrízio, Paulo, Ricardo, Juliana, pelas contribuiçôes incondicionais e por tornarem descontraídas, as tardes no laboratório.

Agradeço também às minhas grandes amigas: Aline, Gisele e Jerusa quıe, apesar da distância e do tempo, não se esqueceram de mim

A todos os professores e funcionários do Instituto de Ciências Matemáticas c de Com- 
putação pela gentileza c atenção diariamente prestadas.

Agradeço ao CNPq polo anxílio financeiro.

Enfim, a todos aqueles que colaboraram de alguma forma para a realização destic trabalho.

Muito Obrigada!

"De tudo ficam três coisas:

A corteza de que estamos sempre começando,

A certeza de que é preciso contimuar,

E a certera de que podemos ser interrompidos antes de terminarmos.

Fazer da interrupçäo um caminho novo.

Da queda um passo de dança,

Do medo ruma escada,

Do sonho uma ponte.

Da procura um encontro."

E assim, terá valido a pena existir...

Fernando Sabino 


\section{Resumo}

Os Ensaios de Proficiência por comparação interlaboratorial tền sido um importante mecanismo para controlar a consistência dos laboratórios. Instituições do governo, como o INMEIRO, têm utilizado tais mecanismos para monitorar a qualidade dos serviços prestados pelos laboratórios da Rede Brasileira de Laboratórios (RBL) e da Rede Brasileira de Calibração (RBC).

Atualmente, os mótodos cstatísticos utilizados para analisar os resultados dos Ensaios de Proficiência estão escritos ern normas técnicas, como o ISO/IEC Guide 43-1. Recentemente, Leão, Aoki e Silva (2002) propuseram um método de regressão para testar a competência dos laboratórios, utilizando a distribuição normal multivariada para modelar os dados e estabelecer testes estatísticos.

Como em medições a presença de valores extremos ć constante, vamos modelar os dados utilizando a distribuição t-student, para acomodar tais valores extremos. Neste modelo, estamos interessados em estimar o grau de liberdade e o parâmetro de tendência da medição do laboratório com respeito ao valor de referência, já que os laboratórios vão ntilizar sistemas de mediçốs similares para medir o mesmo ítem, com incertezas determinadas por um processo de calibração. Fncontraremos os estimadores de máxima verossimilhança e de momentos para estes dois parâmetros e vamos desenvolver um teste para avaliarmos a consistência das medições dos laboratórios. No final, a título de aplicação, vamos analisar os dados obtidos pela REMESP (Rede de Metrologia de São Paılo) na área de eletricidade, onde vamos medir a. tensão DC de um multímetro digital. 


\section{Abstract}

The proficiency essays by interlaboratorial comparison have been an important mechanism to control the consistency of the measurements of the laboratories. Government institutions, such as INMETRO, have used such mechanisms to monitor the quality of the laboratories services supplied by the Laboratories Brazilian Network (RBL) and by the Calibration Brazilian Network (RBC).

Currently, the statistical methods used to analyze the results of the Proficiency Essays are described in technical norms, like the ISO/IEC Guide 43-1. Recently, Leão, Aoki and Silva (2002) proposed a regression method to test the laboratories ability, using the multivariate normal distribution to fit the data and to establish statistical tests.

Like in measurements the presence of extreme values is constant, we are modelling the data using the multivariate t-student distribution, to accommodate such extreme values. In this modol, we are interested in estimating the degrees of frecdom and the tendency parameter of the laboratory measurement relating to the reference value, since the laboratories are using similar measurements systems to measure the same item, with standard deviation determined by a calibration process. We are finding the maximum likelihood and moments estimators for these two parameters and are developing a test to evaluate the laboratories measurements consistency. At the end, we are analyzing the obtained data for the REMESP (São Panlo Metrology Network) in the electrical area, where we are measuring the digital multimeter DC tension. 


\section{Sumário}

1 Introdução 1

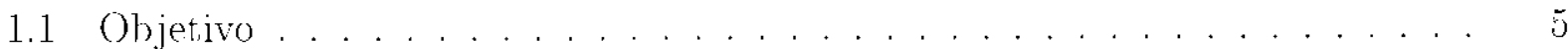

1.2 Organização do trabalho . . . . . . . . . . . . . . . j

2 Apresentação dos dados e Avaliação da Incerteza de Medição $\quad 7$

2.1 Apresentação dos dados . . . . . . . . . . . . . . . . . . . 7

2.2 Avaliação da Incerteza de Medição . . . . . . . . . . . . . . . . . . . . 11

2.2 .1 Equação de Medição . . . . . . . . . . . . . . . . . . . . . 13

2.2.2 Avaliação da Incerteza Padrão de Medição das Estimativas de Entrada 15

2.2 .3 Avaliação da Incertera Padrão Combinada . . . . . . . . . . . . . . 18

2.2 .4 Incerteza Expandida de Medição f . . . . . . . . . . . . . . . . . . . 19

3 A distribuiçāo t-student $\quad 23$

3.1 Introdução . . . . . . . . . . . . . . . . . . . . . . 24

3.2 A distribuição t-student multivariada . . . . . . . . . . . . . . . . . 25

3.2 .1 Propriedades da t-student . . . . . . . . . . . . . 25

3.3 Distribuiçōes relacionadas . . . . . . . . . . . . . . . . . . . 26

4 Modelo Estatístico e Estimaçāo 28

4.1 Modelo proposto . . . . . . . . . . . . . . . . . . . . . . 28

4.2 Estimador para os Parâmetros do Modelo . . . . . . . . . . . . . . . 33

4.2.1 Estimador de Mornentos . . . . . . . . . . . . . . . . . 34

4.2.2 Estimador de Máxima Vorossimilhança (EMV) . . . . . . . . . . . . 34

4.3 Consistência dos estimadores de $\alpha_{i} \ldots \ldots \ldots \ldots$ 
5 Testes de hipóteses $\quad 39$

5.1 Teste individual para os laboratórios. . . . . . . . . . . . . . . . . . . . . . . 39

5.1 .1 Poder do Teste . . . . . . . . . . . . . . . . . . . . 40

5.2 Testando o grupo de laboratórios. . . . . . . . . . . . . . . . 40

6 Aplicaçōes $\quad 42$

6.1 Teste de Grubbs . . . . . . . . . . . . . . . . . . . . . . . . . . 43

6.2 Análise da compatibilidade dos láboratórios . . . . . . . . . . . . . 44

7 Considerações finais $\quad 48$

7.1 Conchusão . . . . . . . . . . . . . . . . . . . . . . . . . 48

7.2 Propostas futuras . . . . . . . . . . . . . . . . . . . . . 49

A Estimador de Momentos para o parâmetro $\nu \quad 50$

Referências Bibliográficas $\quad 52$ 


\section{Lista de Figuras}

2.1 Malha . . . . . . . . . . . . . . . . . . . . . . 8

2.2 Artefato . . . . . . . . . . . . . . . . . 8

2.3 Box-plot dos dados . . . . . . . . . . . . . . . . . . 10

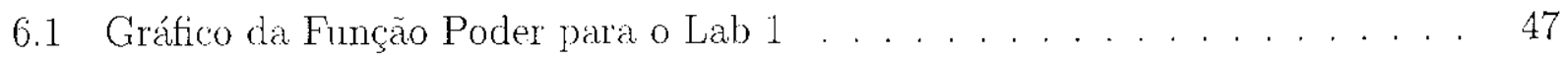

6.2 Gráfico da Função Poder para o Lab 6 . . . . . . . . . . . . . . 47 


\section{Lista de Tabelas}

2.1 Rotciro scguido pelo artefato . . . . . . . . . . . . . . . . 9

2.2 Mediçoes dos laboratórios . . . . . . . . . . . . . . . . . . . . . . . . 9

2.3 Dados do laboratório $5 \ldots \ldots \ldots$

2.4 Cálculo de incertezas para o laboratorio $5 \ldots \ldots . \ldots . \ldots . . \ldots 22$

6.1 Teste de Grubbs . . . . . . . . . . . . . . . . . . . . . . . . . . . . . 43

6.2 Teste para avaliar a competência dos 7 laboratórios . . . . . . . . . . 45

6.3 Cálenlo da Função poder para o Lab $1 \ldots \ldots 6$ 


\section{Capítulo 1}

\section{Introdução}

Um ponto importante no controle de qualidade na indístria é a confiança nos dados obtidos por meio de sistemas de medição. Um sistema de medição ć um processo que envolve instrumentos, operadores e métodos para obter o valor de mua grandeza a ser medida, isto é, o mensirado. Em geral, o valor resultante de uma medição ć somente uma aproximação ou estimativa do valor do mensurado, logo, deve estar acompanhada da incerteza. A incerteza da medição indica quäo correto ć o resultado declarado, isto é, quão corretamente o resultado da medição representa o valor da grandeza que está sendo medida. Para mais detalhes sobre medição e incerteza, ver ISO GUM (1998). Como conseqüência, é importante monitorar a consistência dos laboratórios para realizarem nediçoes específicas.

A REMESP (Rede de Metrologia do Estado de Säo Panlo) é numa entidade que representa, de formáa organizada, os laboratórios de calibraçoes e ensaios junto aos órgãos píblicos e privados, promovendo a integração, o desenvolvimento da competência técnica e da gestão dos laboratórios, visando a credibilidade dos produtos e serviços na busca permanente da competitividade.

A REMESP organizon diversos comitês técnicos para a realização do cnsaios de proficiência. Dentre os comitês já instalados, podemos citar: tempcratura, eletricidade, massa c pressão. Os laboratórios participantes de cada comitê. medem o mesmo padrão e expõcm suds mediçôes para análise. Aqui, analisaremos os resultados do comitê de eletricidade, cujo mensiurando é a tensão DC.

Ensaios de Proficiência (EP) é o uso de ensaios de comparacoes interlaboratoriais para 
determinar o desempenho dos laboratórios para ensaios (ou calibrações) específicos e para monitorar a consistência e comparabilidade dos dados dos laboratórios. Ensaios de comparações interlaboratoriais são conduzidos nãa somente para analisar laboratórios, como também para avaliar métodos e padrões. Os métodos de EP dependem da natureza do ítem ou material sob teste, do método de ensaio em uso e do número de laboratórios participantes. Existem seis tipos de EP, discutidos no ISO/IEC Guide 43-1 (1997), como descritos abaixo:

a. Esquemas de comparação de medição : É onde um único objeto de teste é distribuído seqüencialmente entre os laboratórios participantes. Cada laboratório envia o objeto para o próximo participante ou para o coordenador para manutenção. É comum para comparar padrões de calibração. Os participantes devem apresentar suas medições e as incertezas padröes associadas;

b. Esquemas de ensaios interlaboratoriais : É onde vários objetos de teste com características idênticas (supostannente) são produzidos, misturados, crubrulhados, e distribuídos para vários participantes. Poden ser distribuídos objetos m duplicata para testar a precisão e exatidão dos laboratórios;

c. Esquemas de divisão de amostras : É onde amostras de um produto ou material são divididos em duas. on mais, partes com cada laboratório participante testando uma parte de cada amostra;

d. Esquemas qualitativos : F́ onde os objetos de teste têm características conhecidas e categóricas. Os resultados podem ser avaliados independente de outros participantes;

e. Esquemas de valor conhecido : É onde os objetos de teste têm características conhecidas e quantitativas. Isso também ocorre quando o objeto de teste é fabricado para produzir uma resposta desejada ou conter uma quantidado conhecida de uma substância. Como no esquema qualitativo, não existe necessidade de se comparar os resultados com outros participantes;

f. Esquemas de processo parcial : É onde o teste envolve somente partes definidas de um ensaio ou processo de medição. Isso pode ser feito para testar conformidades em certas 
ações em um laboratório, tais como ajustar uma curva de calibração on interpretar quantidades radiológicas.

Neste trabalho, nos consideramos o primeiro tipo de ensaio, onde um único objeto de teste ó distribuído sceqüencialmente entre os laboratórios participantes e cada laboratório envia o objeto para o próximo participante apresentando os resultados de suas medições e suas respectivas incertezas expandidas para serem stubmetidas à análise. O artefato que circulou entre os laboratórios é um multímetro digital (Figura 2.2).

As técnicas estatísticas usadas para analisar os resultados dos EP precisam seguir três passos básicos, que é comum para todos os programas de EP :

a. Determinar o valor de referência;

b. Fazer a comparação dos resultados;

c. Determinar nma estatística de performance.

Os resultados dos participantes devem ser comparados com os valores ou respostas que melhor demonstrarem competência com o mótodo. Valores de referência podem ser escollidos para avaliar com justiça os participantes e ainda para estimular o acordo entre os laboratórios. Existem uma variedade de métodos comuns para determinar o valor de referência.

Aqui, nós iremos determinar o valor de referência pelo valor de consenso dos participantes, pois este procodimento é muito usado em programas de acreditação com EP de rotina. Neste caso, é importante fazermos uma análise de valores extremos.

Valores extremos podem ter profunda influência nos resultados. Quando a média e desvio padrăo de consenso são usados para estimar a média c desvio padrão do valor de referência, devemos utilizar procedimentos para controlar os efeitos dos valores cxtremos. Aqui aplicaremos o método de Grublss (1969) para identificar valores extremos. Somente os laboratórios em que as mediçöes não são consideradas como valor extremo continuam em nossa análise.

Os resultados dos participantes tal que as mediçóns não são consideradas valor extremo, podem ser transformadas em estatísticas de desempenho. O objetivo é medir a diferença entre o valor de referencia e o valor do laboratório de tal forma que possa ser comparada 
com um critćrio. Atualmente, as análises são feitas via estatísticas que fornecem uma comparação dircta entre o resultado do participante e o valor de referência. Lima estatística de dessempenho comum em programas de comparação de medição, proposta no ISO/IEC Guide 43-1 (1997), é o Erro Normalizado (EN Labiji $)$ :

$$
E N_{\mathrm{Labi \textrm {i }}}=\frac{\left|\bar{y}_{i .}-\mu_{x}\right|}{\sqrt{U_{\text {labi }}^{2}+U_{r e f}^{2}}} \quad i=1, \ldots, K
$$

onde $\bar{y}_{i}$ é a média do laboratório $i$ e $\mu_{x}$ é a média do valor de referencia, $U_{l a b i}$ e $U_{r e f}$ correspondem às incertezas expandidas do laboratório t e do valor de referência, respectivamente. Na seção 2.2 apresentamos um estudo sobre incertezaś.

Esta estatística de performance, descreve a diferença entre o resultado do laboratório e o valor de referencicia, relativo às incertezas dos valores envolvidos na diferença. Neste caso, cada laboratório é comparado com o valor de referência via teste de hipóteses individuais. No entanto, também é interessante estabelecer um teste conjunto para avaliar a performance do grupo de laboratórios participantes. A avaliação conjunta dos participantes tem um peso significativo no conceito do grupo em relação aos orgäos credenciadores.

O erro normalizado testa as seguintes hipóteses:

$$
\begin{cases}H_{0}: & \text { Laboratório compatível } \\ H_{1}: & \text { Laboratório rão compatível }\end{cases}
$$

ou scja, ao fazer o teste individual, se o valor do erro normalizado cair na região de rejeição $\left(E N_{\text {Labibi }}>1\right)$, podemos conchuir que temos evidências para rejeitar $H_{0}$, com nível de confiança de aproximadamente $5 \%$.

Para analisar os resultados do comitê de cletricidade, vamos propor :

a. Um modelo para explicar os dados:

b. Encontrar os estimadores de máxima verossimilhança e de momentos para os parâmetros do modelo proposto;

c. Desenvolver testes para verificar a compatibilidade entre os laboratórios e a competência de cada laboratório individualmente. 
Como em medições a presença de valores extremos é constante, vamos modelar os dados utilizando a distribucicão t-student, para acomodar tais valores extremos. Neste modelo, estamos interessados em cstimar o gran de liberdade o o parâmetro de tendencia da medição $\alpha_{i}$ do laboratório com respeito ao valor de referencia, já que os laboratórios vão utilizar sistemas de medições similares para medir o mesmo ítern, com incertezas determinadas por $11 \mathrm{~m}$ processo de calibração. Encontraremos os estimadores da máxima verossimilhança e de momentos para estes dois parâmetros e vamos desenvolver um teste para avaliarmos a consistência das mediçoes dos laboratórios. No final. vamos analisar os dados obtidos pela REMESP (Rede de Metrologia de São Paulo) na área de eletricidade, cujo mensurado é a tenisão DC.

\section{$1.1 \quad$ Objetivo}

Considere um programa de comparação interlaboratorial com um grupo de $N$ laboratórios. Fstes laboratórios vão medir ım mesmo equipamento padrão, devidamente calibrado por $u$ laboratório reconhecido internacionalmente. Os laboratórios participantes realizam as medições do equipamento padrão e emitem um certificado com os valores medidos e a incerteza associada.

Em nossa análise iremos comparar os resultados dos laboratórios em relação a $11 \mathrm{~m}$ valor de referência e determinar se o grupo de laboratórios estáa com problemas na medição. Estes problemas são devido a falhas nos equipamentos utilizados na medição, operadores que não seguem corretamente os procedimentos de medição entre outros.

Aqui, propomos um modelo estatístico para os dados provenientes do ensaio de proficiência conduzido na área de eletricidade pcla REMESP. Baseado neste modelo, vamos avaliar o grupo de laboratórios participantes.

\subsection{Organização do trabalho}

To próximo capítulo, apressentaremos os dados referentes ao programa de EP (laboratórios participantes com suas mediçóes e o percurso seguido pelo Multímetro Digital) e a metodologia utilizada para avaliarmos a incerteza da medição. 
No Capítulo 3, citaremos algumas características da distribuição t-student e os resultados mais importantes.

No Capítulo 4, vamos definir um modelo para explicar os dados e calcular o Estimador de Momontos e Máxima Verossimilhança para o parânetro de tendência em relação ao valor de referência e para o gran de liberdade.

No Capítulo 5 descnvolveremos um teste de hipótese para testar a competencia e consistência do grupo de laboratórios e individıalmente.

To Capítulo 6 aplicaremos os dados apresentados no Capítulo 2 aos resultados obtidos neste trabalho.

No Capítulo 7 apresentaremos algumas conclusōes e as possibilidades de trabalhos futiuros. 


\section{Capítulo 2}

\section{Apresentação dos dados e Avaliação da Incerteza de Medição}

Neste capítulo, apresentaremos os laboratórios participantes, o roteiro seguido pelo artefato c os dados relativos ao programa de EP conduzido pela REMESP na área de eletricidade.

Além disso, vamos fazcr uma análise descritiva dos dados via um gráfico de "Box-Plot". Vannos conceitular incertezal o mostrar como os laboratórios aplicam este conceito na medição da t.ensão DC, por um multímetro digital, para estimar a variância de suas medições.

\subsection{Apresentação dos dados}

Buscando a comprovação da compctência técnica en medições, vários laboratórios do Estado de São Paulo se compromoteram a participar do programa de comparação interlaboratorial por ensaios de proficiência, conduzido pela REMESP. Em uma comparação interlaboratorial, os diversos laboratórios medem o mesmo cquipamento. No nosso caso, os laboratórios mediram a tensão DC por um Multómetro Digital.

Essas medições foram realizadas utilizando um multímetro do próprio laboratório (multímetro padrão) e um multímetro que circula entre os participantes do EP (artefato).

Para a coletá dos dados, o experimento foi realizado seguindo os seguintes procedimentos:

- Foi montado uma malha, onde instalamos o multímetro do laboratório, o artefato c ıma fonte geradora de tensão; 


\section{Fonte}

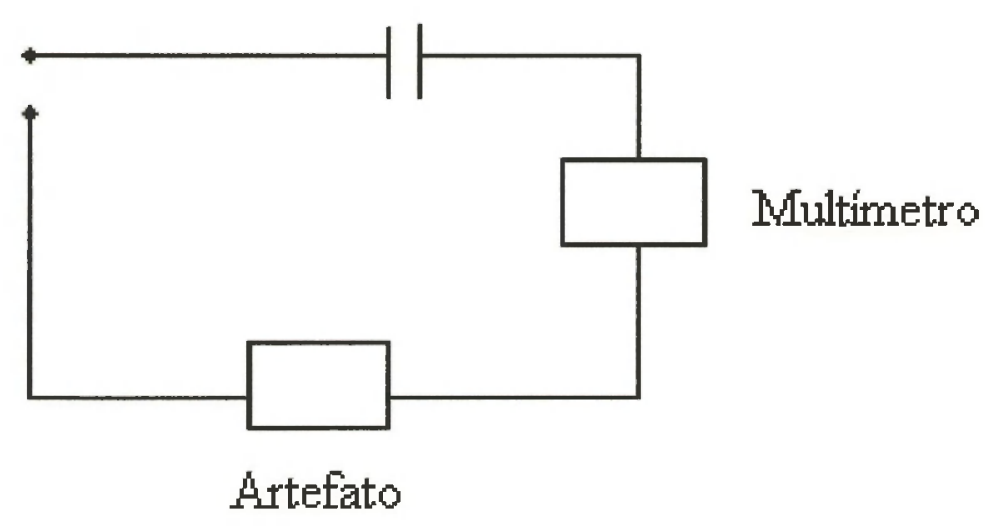

Figura 2.1: Malha

- A fonte foi ajustada para 1 volt, utilizando o multímetro do laboratório;

- A tensão gerada foi medida pelo artefato.

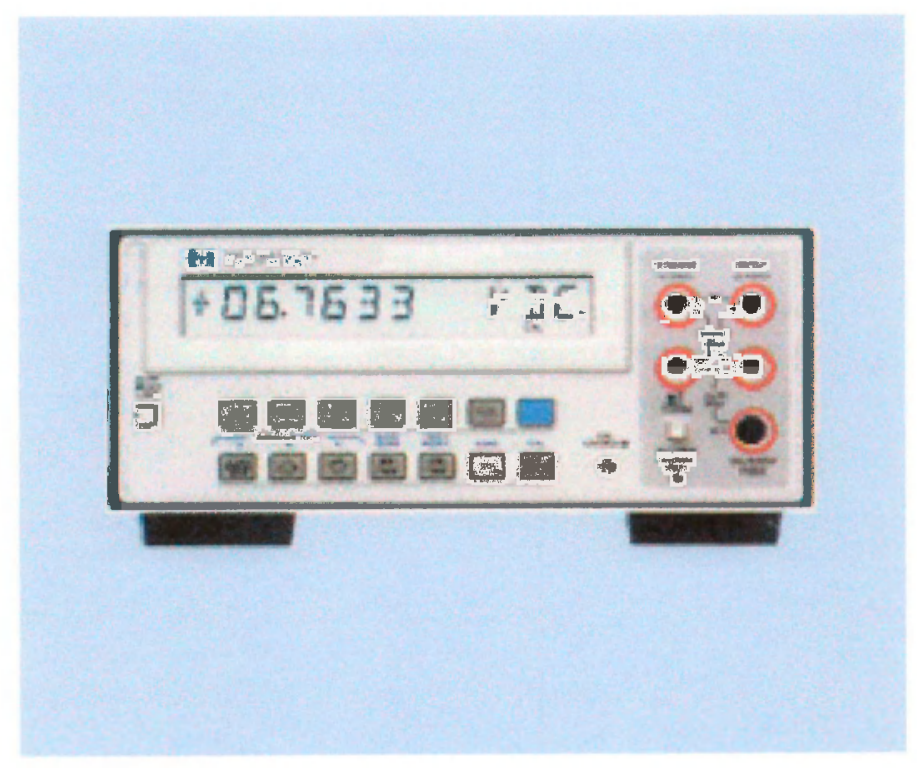

Figura 2.2: Artefato

Os laboratórios participantes do programa e o percurso seguido pelo artefato estão descritos na Tabela 2.1: 


\begin{tabular}{|c|c|c|}
\hline Roteiro & Laboratório & Cidade \\
\hline \hline 1 & STAVALF & Vila Formora \\
\hline 2 & CPDHA & Guarulhos \\
\hline 3 & BALITEK & Ipiranga \\
\hline 4 & MFC-Q & Santo André \\
\hline 5 & SOCINTHC & Ijoiranga \\
\hline 6 & FSFERA & Santa Bárbara \\
\hline 7 & QLALIBRÁS & Campinas \\
\hline 8 & GERO & São Bernardo \\
\hline
\end{tabular}

Tabela 2.1: Roteiro seguido pelo artefato

\begin{tabular}{|c|c|c|c|c|c|c|c|c|}
\hline Leituras & Lab1 & Lab2 & Lab3 & Lab4 & Lab5 & Lab6 & Lab7 & Lab8 \\
\hline 1 & 1.000005 & 0.9999 & 0.99906 & 1,00000 & $1,0(0) 000$ & 0.9999 & 0.99999922 & 1 \\
\hline 2 & $1.0(0) 11$ & 0.9999 & 0,9996 & $(0,999999$ & 1,00000 & 0.9999 & $1,00(10100000$ & 1 \\
\hline 3 & 1.000005 & $1.00(00)$ & 0,9996 & 0,9999 & $1 .(0)(0)(0)$ & 0.9999 & 1,00000002 & 1 \\
\hline 4 & 1,00012 & 0,9999 & 0,9996 & $1,00(0)(\mathrm{J}$ & $1.00(00)$ & 0.9999 & $1,00(0)(0) 11$ & 1 \\
\hline 5 & $1.0(0) 005$ & 0,9999 & 0,9996 & 1,00000 & $1.00(0)(0)$ & 0.9999 & 1,00000012 & 1 \\
\hline 6 & $1 .()(0) 11$ & 0,9999 & 0,9990 & $1,(0)(0) 0)$ & $1.0(0)(0)$ & 0.9909 & 1.0000002 & 1 \\
\hline 7 & 1.00006 & $(0,0999$ & 0,9996 & 1,000000 & $1.00(000)$ & 0.9999 & $1,000(00)^{2} 21$ & 1 \\
\hline 8 & 1.000011 & 0,9999 & 0,9996 & 1.00000 & 1,00000 & 0,9999 & 1,00000023 & 1 \\
\hline 9 & 1,000006 & 0,9999 & 0,$9996 ;$ & 1.00000 & 1,00000 & 0,9999 & 1,00000061 & 1 \\
\hline 10 & 1,00011 & 0,99999 & 0,9996 & 1,00000 & 1,00000 & 0,9990 & $1,0(0)(0)(071$ & 1 \\
\hline Néliat & 1,000008 & 0.999091 & 0.99960 & 0,09998 & 1,0000000 & 0.99990 & 1,000000 & 1.000000 \\
\hline 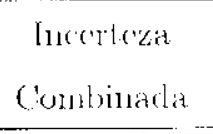 & 0.000001010 & 0,0000037 & 0.000120 & $0,0000760)$ & $01,00(0) 00.11$ & 0,00000008 & 0,00001029 & 0,000120 \\
\hline $\begin{array}{l}\text { Fator do } \\
\text { Abrangencia }\end{array}$ & 2 & 2 & 2 & 2 & 2 & 2 & 2 & 2 \\
\hline
\end{tabular}

Tabrala 2.2: Mediçoes dos laboratórios

Obs.: A sequência dos laboratórios na Tabcla 2.2, não estáa seguindo a mesmá sequência da Tabela 2.1, ou seja, por questão de ética, não vamos identificar as medições dos laboratórios. 
A incerteza combinada (desvio padrão) apresentada na l'abela 2.2 , é o parâmetro que caracteriza a dispersão dos valores que podem ser razoavelmente atribuidos à tensão DC no artefato (ver seção $\mathbf{2 . 2 . 3}$ ). O fator de abrangência é uma constante que multiplicada pela incerteza combinada, fornce um intervalo com $95 \%$ de confiança (ver seção 2.2.4).

Na prática, os laboratórios utilizam características físicas do método de calibração para estimarem o desvio padrào das mediçoes (incerteya combinada). Com o objetivo de estabelecer um intervalo com $95 \%$ de confiança para o resultado da medição, os laboratórios multiplicam a incertera combinada $\left(l_{c}\right)$ pelo fator de abrangencia $k$. Com isso, o resultado da modição é interpretado por

$$
R M=\text { Média das mediçòes } \pm U_{E},
$$

onde $U_{E}=k \cdot U_{C}$ é denominada incerteza expandida que possibilita identificar uma extensa fraça $(95 \%)$ da distribuição de valores que poderiam ser razoavelmente atribuidos ao mensurado. Em geral, a distribuição normal é utilizada para determinar o fator de abrangência. Maiores detalhes scrão discutidos nas seções seguintes.

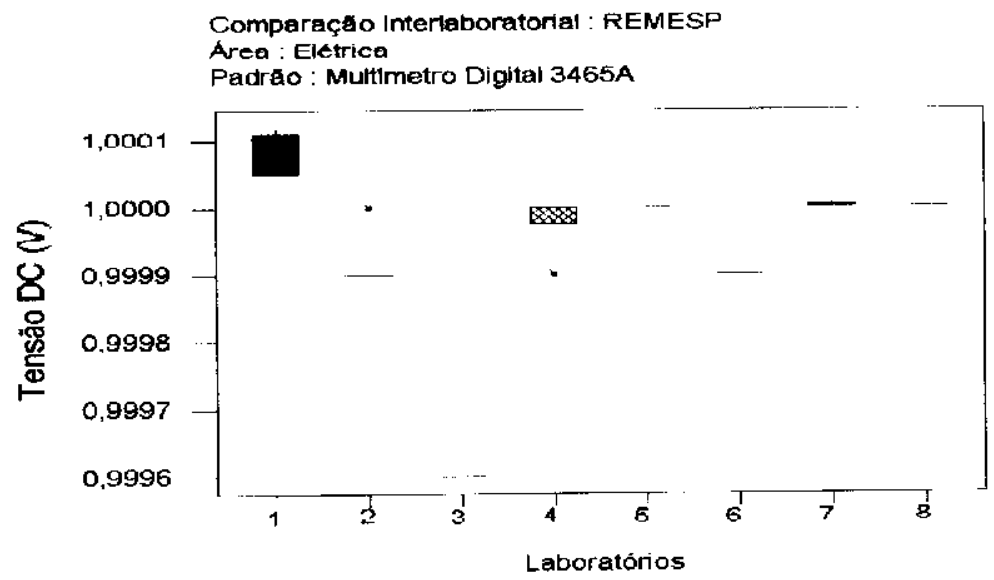

Figura 2.3: Box-plot dos dados

Através do Box-Plot, Figura 2.3, notamos que o laboratório 3 apresenta inedições bem abaixo dos demais laboratórios. Pelo teste de Grubbs (1969), ele foi considerado valor extremo e por isso será excluído da análise. O teste de Grubbs (1969) será discutido no Capítulo 6. 


\subsection{Avaliação da Incerteza de Medição}

O objetivo de nma mediçáo é deteminar o valor do mensirado, isto é, o valor da grandeza esperéfica a ser medida. Una medição conneça, portianto, com mma especificação apropriada do mensurado, do método de medição e do procedinento de medição.

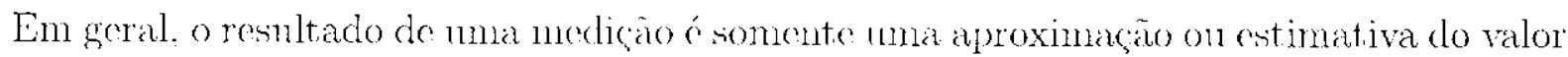
do mensurado e assime só é completa quando acompanhada pela declaragäo da incerteza dessa estimativa.

Na prática, a especificação ou definição do mensurado é ditada prla exatidão requerida da medição (gran de concordâneia entre o resultado de uma medição e nm valor de referência para o mensurado). () monsurado dre ser definido com totalidade suficiente relativa à exatida requerida, de modo que, para todos os fins práticos associados com a medição, senu valor seja único.

A operarçà̃o de medição é realizada, genericannente, por $1 \mathrm{~m}$ sitema de medição (SM). O

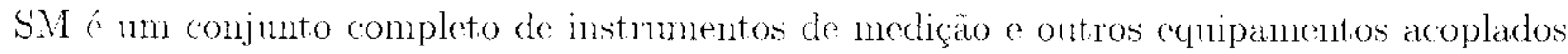
para executar una mediçäo nepeecifica, on seja. é um processo que combina instrumentos, operadores e métodes para obter o valor de mua quantidade a ser medida. Nenhnum sistema de medição é perfeito, on sejar, na realizaçào de mediçoes existem várias influéncias que geran erros nos resultarlos obtidos. de modo que não existo nua mediçäo perfoita (sem erros).

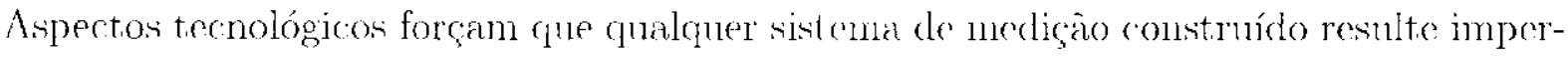

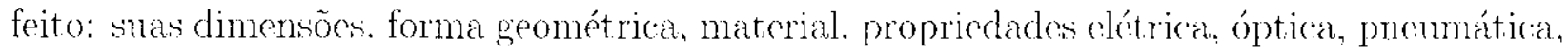
etc. màn correspondem exatamente ao ideal. As leis e princípios físicos que regen o funcionamento der alguns sistomas do mediçäo nom sempre são perfeitamente lineares, como uma análise simplista poderia supor. A existência de desgaste o deterioração de partes agravam ainda mais esta condiçäo. En todos os casos, o SM gera crros de medição.

O erro de medição é impossível de ser elininado, porém podemos ao menos delimitá-

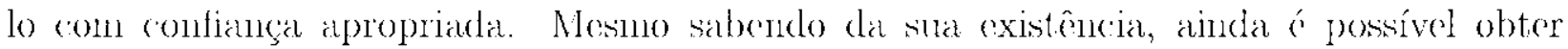

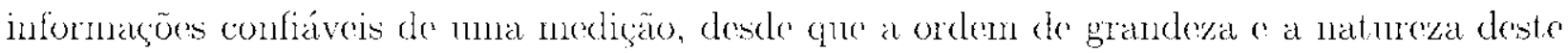
erro sejam conhecidas. O erro ó nun conceito idealizado e näo pode ser exatamente conhecido.

Geralnente ocorrem erros de vários tipos em numa mesina medida. Entretanto, essess diferentes tipos de erros porlem ser separados em trés grandes grupos: 
1 Erros Aleatórios ou Erros Estatísticos: Resulultan de variações aleatórias no valor medido de uma grandeza, devido a fatores que não podem ser controlados on que por qualquer motivo não säo controlados. Geralmente. cssas variaçóes se devem somente ao sistema e processo do medição. mas en algnus casos as variaçós aleatórias também ocorrem 1 a própria grandezad. Diversos fatores contribuem para o surgimento dos crros aleatórios, por exmplo, existência de folgas, vibraçồs, flutuaçós de tensão elétrica, instabilidades internas on das condiçoes ambientais, etc...

2. Erros Sistemáticos: É a parcela de erro sempre presente nas medições realizadas sob condiçoes de repetitividade. Da mesma forma que os crros alcatórios, os erros sistemáticos näo podem ser totalmente eliminados, mas podem ser reduzidos através de correções para compensar os seus cfeitos. Os erros sistemáticos mais aplicados säo:

2.1 Erros Sistematicos Instrumentais: F́ o erro que resulta da calibraça de um instrmunento de mediçào.

2.2 Erros Sistemáticos Tróricos: É encontrado no 12 so de fómulas teóricas aproximadas on 11 so do valores aproximados para eventuais constantes físicas que sejam utilizadas.

2.3 Frros Sistemáticos Ambientais: É o erro cansado devido a efeitos do ambiente sobre a medição, tais como, temperatura, pressãa,o, umidade, aceleração da gravidade, elc...

2.4 Erros Sistemáticos Observarionais: É o enro devido a falhas on limitação do próprio operador.

2.5 Frros Sistemátiós Residnais: São os erros que não podem ser reduzidos a 110 valor baixo on para os quais não seja possível fazer correçòs.

3 Frros Grosseiros: Consiste de cmganos que. "vent lalmente podem ocorrer no procedimento de medida on nar realizaçào de cálenlos.

Na grande maioria dos casos, o resultado da nediçäo é determinado através de mma série de leiturat obtidas sob condiçoes de repetitividade. Variaçoes obtiklas mas leituras repetidas sào consequîneias de fatores que afetam os resultados das leituras. Além disso, o modelo 
matcmático da medição, que transforma as leituras repetidas no resultado da medição é crítico, pois inclui fatores que năo são totalmente conhecidos. Assim, a variação obtida nas leituras repetidas a a falta de informaçào do modolo matemático, contribuem para a incerteza do resultado da mediçăo.

A declaração do resiltado de muna medição somente é completa se cla contiver tanto o valor atribúclo ao mensurado quanto a incerteya de medição associada a este valor. A

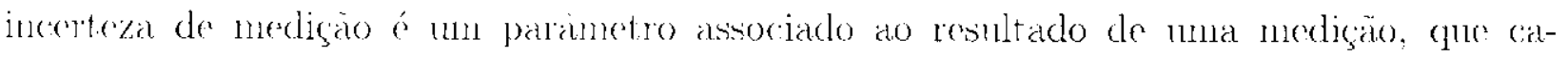
racteriza a dispersio dos valores que podem ser razoavelnente atribuídos ao mensurado (ISO/GL M(1998)). O mensmado ó a grandeza física a ser medida. En geral os passos para rstimar a incerteza săo:

a. Definir a equacăo de medição;

b. Identificar as componentes de incerteza;

c. Catcular a incertezal padrào das componentes;

d. Calcular a incertege combinada;

e. Calcular os grans do liberdade efetivo:

f. Calcular a incertera expandida.

Nas subseções segnintes, abordaremos cada passo mencionado acima.

\subsubsection{Equação de Medição}

Na maioria dos casos o mensurado Y não é medido diretamente, mas é determinado a partir de $X$ outras grandezas $X_{1}, X_{2}, \ldots X_{N}$ através de mon relaçäo funciomal f:

$$
Y=f\left(X_{1} \cdot X_{2}, \ldots X_{N}\right)
$$

As grandezas de entrada $X_{1}, X_{2}, \ldots X_{N}$, das quais a grandeza de saida $Y$ depende, podem ser consideradas como: 
1 Grandezas cujos valores e incertezas polem ser diretamente determinadas na medição em curso. Fstes valores on incertezas poclem ser obtidos, por exemplo, de nma rinica obscrvaçăo, de observaçôs repetidas on de julgamento baseado na experiência, c podem envolver a determinaça de correçöes a Inituras de instrumentos e correçoos por conta de grandeza de influência, tais como temperatura ambiente, pressão barométrica $\mathrm{c}$ iumidade:

2 Grandeyas cujos valores a incertezas são incorporados à medição a partir de fontes externas, tais como grandezas associadas com padrões de mediçäo calibrados, materiais de referencia cortificados e dados de referencia obtidos de manuais técnicos.

No progranna de chetricidade. como método de nedição. cada laboratório muilizon a seguinte relaçäo:

$$
M_{A}=\Delta+M_{P}+\operatorname{Res}(A)+\operatorname{Res}(P)
$$

onde,

- $M_{11}$ : Representa a tensão no artefato:

- $\Delta$ : Repetitividade;

- Mp: Representar a lensäio no ecpuipanento padrão do laboratório:

- Res $(\Lambda)$ : Resolução do artefato:

- Res(P): Resolução do equipamento padrão do laboratório.

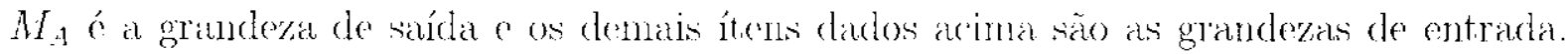

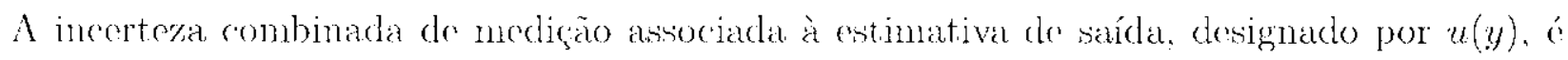
o desvio padrão do mensirado Y o deve ser determinado a partir das estimativas $x_{;}$das grandezas de entrada $X_{i}$ e suas incertezas padrão associadas $u\left(x_{i}\right)$. Abaixo descreveremos métodos para calcular a incerteza de cada grandeza de entrada. 


\subsubsection{Avaliação da Incerteza Padrão de Medição das Estimativas de Entrada}

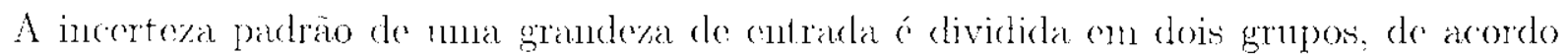
com o método utilizado para cstimar senes valores muméricos:

- Tipo A: Aquelas que säo avaliadas por métodos estatísticos;

- Tipo B: Aquelas que são avaliadas por ontros métodos.

Independente de suas classificaçòes, todos os componentes de incertera são modelados por nuna distribuição de probabilidade e cuantificados pela variância ou desvio padrão. A incerteza padrão do Tipo A é obtida a partir de unia finção densidade de probabilidade

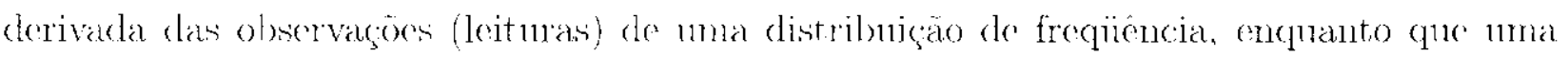

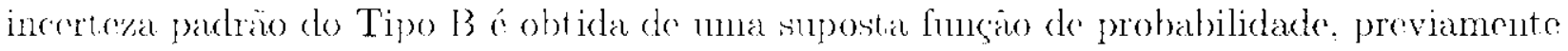

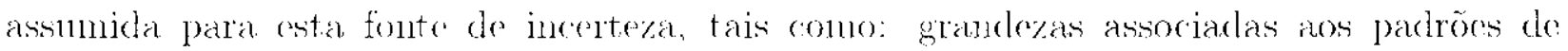
merliçào calibrados, materiais de referencia con certificardos e ontross.

É importante ressaltar que toda inecrte\%a padrão á nun desvio padrão. Assirn, se considerarmos a incerteza do tipo A tremos o desvio padrão da média da série de observações da medição. Por outro lado, se consideramos as incertezas do tipo B, temos o desvio padrão da distribuceço de probabilidado associada.

A incerteza padrão das grandezas de entrada $\Delta$ é avaliada pelo tipo A encuanto que $M_{L}$. $\operatorname{Res}(P)$ e $\operatorname{Res}(L)$ são avaliadas pelo tipo [3. Como fonto de incerteza do tipo A, consideramos a repetitividade clas nuediçons. No nosso caso, cada laboratório realizon dez leituras da lensão DC. Para exemplificar os cálculos, vamos tomar os dados clo laboratório 5. 


\begin{tabular}{|c|c|}
\hline Isituras & Trnsä́o DC $(\mathrm{V})$ \\
\hline \hline 1 & 1.00000 \\
\hline 2 & 1,0000 \\
\hline 3 & 1,00000 \\
\hline 4 & 1,00000 \\
\hline 5 & 1.0000 \\
\hline 6 & 1,0000 \\
\hline 7 & 1,00000 \\
\hline 8 & 1.0000 \\
\hline 9 & 1.0000 \\
\hline 10 & 1.00000 \\
\hline \hline Média & 1.00000 \\
\hline DPM & 0 \\
\hline
\end{tabular}

Tabela 2.3: Dados do laboratório 5

A incerteza padrão das grandezas de entrada é estimada da seguinte forma:

- Incertczas Avaliadas pelo tipo A: Apui, a incertewa padrào é dada pelo desvio padrão da média da série de observaçös da medição de telsăo. Sejam $t_{1}, t_{2}, \ldots, t_{10}$ as de\% mediçons da tonsào (ver tabela 2.3). O desvio padräo da módia é

$$
S(t)=\frac{S(t)}{\sqrt{10}} \cdots: 0
$$

onde $S(t)=\sqrt{\frac{1}{9} \sum_{k=1}^{10}\left(t_{k}-\bar{t}\right)}=0 e \bar{t}=\frac{1}{10} \sum_{k=1}^{10} t_{k}=1$. Portanto, a incerteza padräo da repetitiviraclé

$$
u(\Delta)=0
$$

- Incertezas Avaliadas pelo tipo B: $\Lambda$ qui, a incerteza padrão de mad grandeza de entrada que não tenla sido obtida através do observaçoes repetidas é avaliada por julganento científico, bascando se mu todats as informagoes dimponiveis sobre a possível variabilidade dessa grande\%ar. O conjunto de informaçons pode inchin: 
1. dados de medições prévias;

2. a experiencia on o couhecimento geral do comportamento e propriedades de mat.riais c instrumentos relevantes:

3. especificaçons do fabricante;

4. darlos fornecidos em certificados de calibração e outros certificados;

5. incerteras relacionadas a dados de referência extraidos de mamnais.

A partir disso. e considerando a grandezas de entrada para a medição da tensão, as

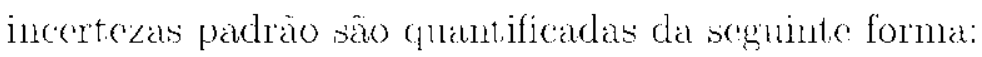

- Incerteza Padrão para $M_{P}$ : O desvio padrão da tensão no padrão do laboratório năo é estimado da variabilidade associada diretamente às medições mas via a incerteza expandida herdada do multímetro. Como veremos na segào 2.2.4 a incerteza expandida é $u m$ múltiplo do desvio padrão que abrange o semi-intervalo de $95 \%$ de probabilidaule. Assim, utilizando os dados da tabela 2.3, obtemos que:

$$
0,000013=2 * u\left(M_{P}\right) \Rightarrow u\left(M_{P}\right)=0,0000065
$$

Portanto, a incerteza padrão do padräo do laboratório é u( $\left.M_{P}\right) \cdots 0,0000065$.

A incerteza expandida do equipanent o padrão do laboratório está declarada no certificado de calibração do respectivo equipamento.

- Incerteza Padrão para Res(A): Para esta grande\%a temos a informação qun o

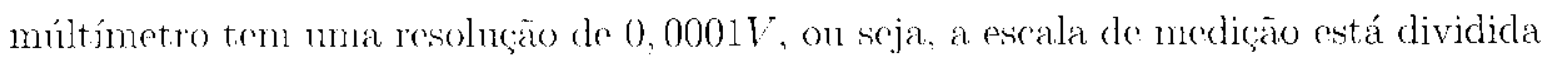
('n intervalos de $0,0001 \mathrm{~V}$. Sendo assim. vamos considerar que Res( $A$ ) tem distribuição nuiforne entre estes intervalos. Portanto, a incertevia padrão da Res $(A)$ ó:

$$
u(\operatorname{Res}(A))=\frac{0,0001}{\sqrt{12}}=0,0000289
$$

- Incerteza Padrão para Res(P): Assin como Res(A). Res(P) também é consideradia

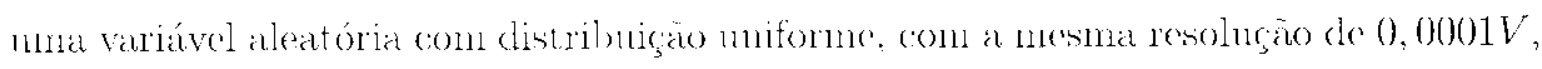
$\log 0$, 


$$
u(\operatorname{Res}(P))=\frac{0.0001}{\sqrt{12}}-0.0000289
$$

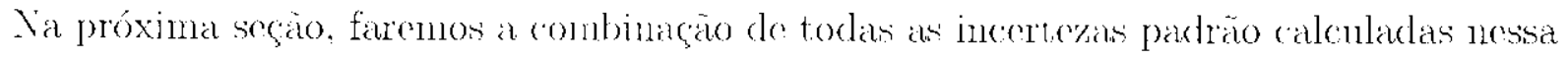
seção.

\subsubsection{Avaliação da Incerteza Padrão Combinada}

A incertera combinada é a combinagão entre todas as incertezas padräo das componentes relacionadas pela equação de medição. on soja, uc(y) corresponde ao desvio padrão dá estimativa da grandeza de saída.

Em alguns estudos de calibração. podemos ter que duas on mais grandezas de entrada

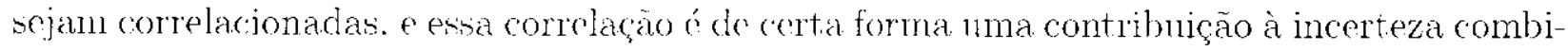
llada. Para julgar se cxiste ou não essa correlação é preciso conhecer o processo de medição e ter conhecimento de causa dessa dependência nuútna entro as grandezas de entrada. Não tonar conhecinnento dessisas correlações pode levar a avaliações incorretas da incerteza padrão do mensirado.

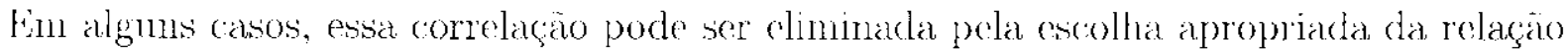
funcional, mass na prática, algnnnas grandezas sào frequentemente relacionadas pois, na aval-

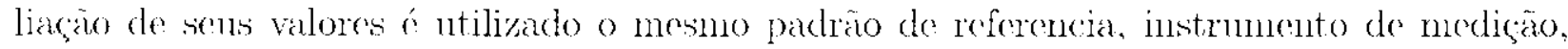
dados de referencia, on até, o método de medicáo.

Vos casos onde existe a correlação, o quadrarlo da incerteza padrão combinada (ISO) GLM, 1998, pg. 21. item 5.2.2) podo ser aproximado por.

$$
u_{c}^{2}(y)=\sum_{i=1}^{N} c_{i}^{2} u^{2}\left(x_{i}\right)+2 \sum_{i=1}^{N-1} \sum_{h=i+1}^{N} c_{i} c_{h} u\left(x_{i}, x_{h}\right) .
$$

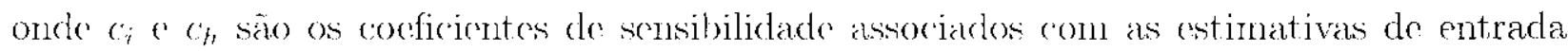
$x_{i}$ e $x_{h}$. respectivamenté isto é as derivadas parciais da relação finncional $f$ com relaçăo às variáveis $X_{i} \circ X_{h}$, avaliadas para as estimativas $x_{i}$ e $x_{h}$.

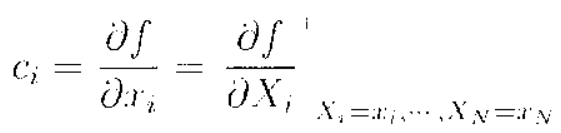

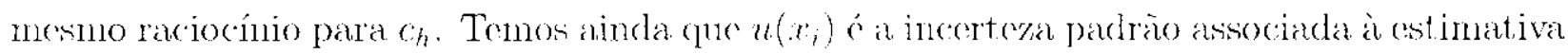
$x_{i}$ da grandeza de entrada $X_{i}$ (avaliada conforme seçĩo anterior) o $u\left(x_{i}, x_{h}\right)$ é a covariáncia 
associada às duas estimativas $x_{i}$ e $x_{h}$ dada por

$$
u\left(x_{i}, x_{h}\right)=u\left(r_{i}\right) u\left(x_{h}\right) \times\left(x_{i}, x_{h}\right) \quad(i \neq h)
$$

onde, $r\left(x_{i}, x_{h}\right)$ é o cocficiente de correlação $(t \neq h$ e $|r| \leq 1)$. Em alguns casos a covariância pode ser estimada por

$$
u\left(x_{i}, x_{n}\right)=\frac{1}{n(n-1)} \sum_{j=1}^{n}\left(x_{i j}-\bar{x}_{i}\right)\left(r_{h j}-\bar{x}_{h}\right) .
$$

onde $x_{i j}$ ć a j-ćsima observação da grandeza $X_{i}$ e e $\bar{x}_{i}$ é a estimativa da grandeza $X_{i}$, mesmo raciocínio para $x_{h j},(i, h=1, \cdots, N)$.

Para grandezas de entrada não correlacionadas, como ó o caso do grupo da área de temperatura, a incerte\%n combinada é dada por :

$$
u_{i}^{2}(y)=\sum_{i=1}^{N} c_{i}^{2} u^{2}\left(x_{i}\right)=\sum_{i=1}^{N}\left(c_{i} u\left(x_{i}\right)\right)^{2}=\sum_{i=1}^{N} u^{2}\left(x_{i}\right) .
$$

() coeficiente de sensibilidade cy descreve o puanto a estimativa de saída y é influenciada por varlaçós da estimativa de entrada $x_{i}$. Conno em nossá equação de mediçäo a grandeza de suída é uma função linear das granclezas de entrarla, temos qute, para todas as grandezas de entrada, $c_{i}-1$.

Logo, utilizando os cálculos das inecrteyas das grandezas de entrada da seção anterior, obtemos que:

$$
u_{c}^{2}(y)=(0)^{2}+(0.00000060)^{2}+2 *(0,0000289)^{2}
$$

Assim,

$$
u(y)=0,0000) 1138
$$

\subsubsection{Incerteza Expandida de Medição}

Embora a incerteza combinada $u_{c}(y)$ possa ser mivermalmente usada para expresisar a

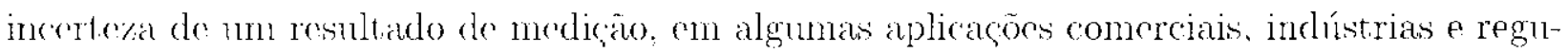
lamentadoras, e quando a saúte e a segurançà estão en cunestão, é muitas veres necessário 
dar uma medida de incerteza, que defina um intervalo en torno do resultado da medição com o qual se espera abranger uma extensa fração da distribucção de valores que poderiam ser razoavelmente atribuídos ao mensurado.

A medida de incerteza que salisfaz o requesito de fornecer tal intervalo é denominada incerteza expandida é é representada por $u_{e}(y)$. Assimn o resultado de uma nedição ó representada por

$$
y \pm u_{\epsilon}(y)
$$

A incerteza expandida $u_{e}(y)$ é obtida nultipicando-se a incertéta padrão combinada $u_{c}(y)$ por $u$ fator k de abrangência, correspondente a uma probabilidade de cobertura especificada (ISO/GUM recomenda 95\%). logo, a expressão acima pode ser rescrita como sendo

$$
y \perp k: * u_{c}(y)
$$

A combinaçào entre as grande’as de entrada cstabelece, pelo Teorema Central do Limite. que a distribuição do menssurado $Y$ será aproximadanente normal. No entanto, podemos ter em algunis (asos, que a incerteza padräo combinada pode estar sendo dominada por componentes de incerteras padräo avaliadas pelo tipo A, baseada em pouras observaçós. on por componentes de incertezas padräo avaliadas pelo tipo 13, baveadas em numa suposta. distribuição não normal. Com ission, ao invós de manmos o valor de ti da distribuição nommal, podennos mellhorar a aproximação observando a distribuceçüo da costatística.

$$
T=\frac{y-Y}{\sqrt{\operatorname{Vur}(Y)}}=\frac{y-Y}{\sqrt{u_{c}^{2}(y)}}=\frac{y-Y}{u_{c}(y)} .
$$

onde $u_{c}(y)$ ó a costimativa para o desvio padrão da grandeza de saída $Y$.

Pelo método de Satterthwaite, a estatística $T$ tem distribuição aproximadamente t-Student com $\nu_{\text {eff }}$ grans de liberdade. O método utilizado estima os graus de liberdade bascado nos grans de liberdade de cadia fonte de incertewa (ISO/GUM,1998, pg 64), na forma

$$
\nu_{\mathrm{eff}}=\frac{u_{i: 1}^{1}(y)}{\left.\sum_{i-1}^{N} \underline{u}_{i}(y)\right)^{4}}
$$

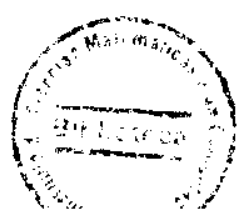


onde os $u_{i}(y)=c_{i} u_{i}\left(x_{i}\right)$ com $i=1, \cdots . N$ são as contribuções para a incerteza padrão associada a estimativa de sajda y. resultante la incerteza padrão associada à cstimativa de crutrada $x_{i}$ e e $\nu_{i}$ săo os graus de liberdade efetivo da contribuiçăo da incerteza padrão $u_{i}(y)$.

Como a estatística $T$ tem distribuição aproximadamente t-Student, o intervalo com nívol de confiança $(1-\gamma)$ para o mensurado $Y$ é dado por

$$
P\left(-t_{2} \leq T \leq t_{\frac{1}{2}}\right)=(1-\gamma)
$$

011 seja,

$$
-t_{\frac{1}{2}} \leq \frac{y-Y}{u_{c}(y)} \leq t_{\frac{i}{2}} \Rightarrow y \pm l_{\frac{\gamma}{2}} u_{c}(y)
$$

onde $l_{\frac{\bar{y}}{2}} e_{0}$ o quantil $\left(1-\frac{\gamma}{2}\right) * 100 \%$ da distribuiçăo t-Student que depende dos graus de librrdade dados por (2.1). Em geral, se denota $k$ (Fator de Abrangência) ao invés de $t_{\frac{2}{2}}$.

Para os dados da tabela 2.3, temos que o gran de liberdade ó dado por

$$
\nu_{\text {eff }}=(0.00004138)^{4} *\left(\frac{0^{1}}{9}+2 * \frac{(0,0000289)^{1}}{30}+\frac{(0,0000065)^{1}}{30}\right)^{-1} \approx 62,966 .
$$

Obs: Para o cálculo de $\nu_{\text {eff }}$ temos que os grans de liberdade com relagäo à repetitividade é iglual a 9 (já que foram roalizadas 10 mediçoes en cada patamar de temperatura). Para

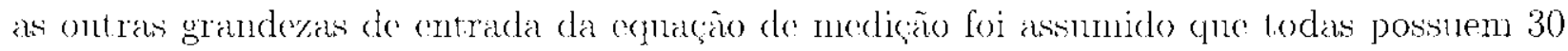
granis de liberdade, conforme recomendação do INMETRO.

Arredondando para baixo (sempre), temos $\nu_{\mathrm{el}}=62$. Considerando um nível de confiança de $95 \%$ oblonos da distribuição t-student que $k \approx 1,999$ e então, a incerteza expandida dacla por

$$
u_{e}(y)=k \cdot u_{c}(y)=1,999 * 0,00004138=0,00008271 \mathrm{~V} .
$$

Resumiremos os cálculos de incertèa apresentados para o laboratório 5 em forma de tabela. 


\begin{tabular}{|c|c|c|c|c|c|c|}
\hline \multicolumn{7}{|c|}{ 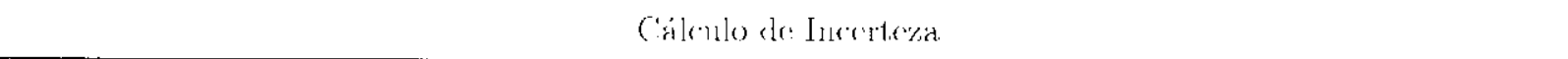 } \\
\hline Fonte de Incerteza & Estimativat & $\left.T i_{p}\right)$ & Distribuicaño & Divisol & Inecerteza & G.L \\
\hline liepertitividade: & () & A & Nurinal & 1 & () & 9 \\
\hline 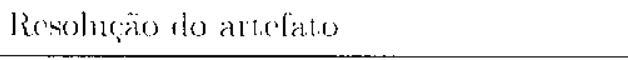 & 0.0001 & [3 & R('limgnglatr & 3,1611 & $0,00(000289$ & 30 \\
\hline 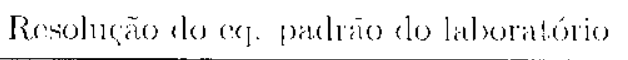 & $0.0(x) 1$ & {$[3$} & Rovarugulate & 3,1611 & $0,000(00289$ & 30 \\
\hline Iucertema do cop padrão do laboratóno & 0.00000133 & $\Gamma 3$ & Normal & 2 & 0,00000065 & 30 \\
\hline Inecrteza Combinarla & & & & & 0,0000 & 31138 \\
\hline Gran de Liberelate lefetivo & & & & & & 2,966 \\
\hline Fator de $\Delta$ brangencial & & & & & & 1.999 \\
\hline Incorteca Expardida & & & & & 0,0000 & 8271 \\
\hline
\end{tabular}

Tabela 2.4: Cálenlo de incerterasis para o laboratório 5 


\section{Capítulo 3}

\section{A distribuição t-student}

As boas propriedades da distribuiçào normal (univariada e multivariada) juntamente com o fato de que a normalidarle pode ser habitualnnente justificada apelando ao Trorema Central do Limite, têm sido as findamentações básicas da aplicação do modelo normal na anćlise estatística de dados contímos. Assim. como a bem sabido, a maior parte da teoria de inferêneia cstatística para variáveis reais contínuas está desenvolvida em torno deste modelo. Este desenvolvimento encontra-se particularmente enfatizado no contexto dos modelos linnares. Contudo, chn multas situaçoos este modelo é claramente impróprio, por exemplo, quando os dados provém de uma distribuigão com caudas mais pesadals que a distribução normal. De fato. a vulnerabilidade da inferencia baseada no nodelo normal com respeito a valores extremos ("outliers") tem sido motivo de vários cstudos de robuste\%. Fin particnlar, a deteçäo e tratanento de valores extremos tim representado ma importante área de pescquisa da estat ística robusta. Nesite sentido, alguns modelos alternativos ao nomale que säo bascados em distribuiçöes simétricas que perniten reduzir a influência dos valores extremos têm sido sugerido por diterentes antores. Por exemplo. Taylor, Little o Lange (1989) propoom o modelo t-student com $\nu>0$ grans de liberdade como uma extensão paranétrica robusta do modelo normal e ilustram o desempenho do novo modelo em várias aplicações.

Como nossos dados são obtidos de sistemas de mediģno, que estäo sujeitos a erros, a presença de valores extremos "é natural". Desta forma vamos modelar os dados utilizando esta distribuiçäo, encontrar os estimadores de momentos e máxima verossimilhança para os parâmetron (ver Capítulo 4) e desenvolver testes de hipóteses (ver Capitulo 5). Neste. 
capítulo vamos listar suas propriedades e alguns resultados importantes que serăo usados posteriormente.

\subsection{Introdução}

Nas tress últimas décadas, tem sido observado nun crescente interesse pela distribuição t-student n-variada na literatura c'statística, taulo en teoria como em aplicaçoes. Este

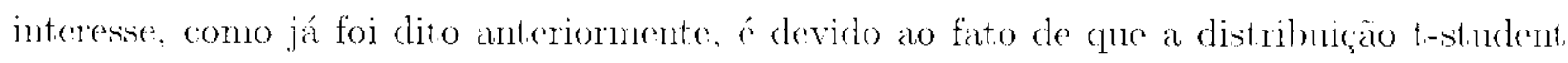
n-variada tôn candas mais pesadas que a clistribuição normal. Além disso, a distribuição t-student aproxima-se da distribuição nomal guando sou gran de liberdade tende ao infinito $(\nu \rightarrow \infty)$.

A distributição t-student multivariada é um membro particular dentro da classse dass distribuiçoes clípticas. De forma geral. dizemos clue a distribuição elíptica é uma extensão da normal n-variada com vetor de médias $\underline{\mu}$ e matriz de covariâncias $\Sigma$, denolada por $N_{n}(\underline{\mu}, \Sigma)$ e a distribuição esférica. uma sub-classe da distribuiçáo elíptical, ó mua classe geral de distribuiçoes con a mesma simetria da distribuiçäo normal padrão n-variada $N_{n}(\underline{0}, I)$. Fm síntese, a distribuiçào elíptica ć uma transformação linesar de locação e escala da distribuiçäo esférica.

Definição 3.1 Seja $O_{n}$ o conjunto das matrizes $\Gamma(n \times n)$ que são ortogonais. ou seja, $\Gamma^{\prime} \Gamma=$

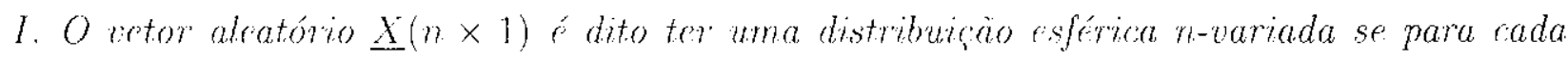
matriz $\Gamma$ em $\mathrm{O}_{n}$. tem-se que

$$
\underline{X}=\Gamma \underline{X} .
$$

O (exemplo mais simples de distribuição esférica é a $N_{n}(\underline{0}, I)$.

Satbemos que a distribuiçăo $N_{n}(\underline{\underline{n}}, \Sigma)$ pode ser escrita om temos da normal $N_{n}(\underline{0}, I)$ da seguninte maneira:

$$
\underline{X}-\underline{\mu}+A^{\prime} \underline{Y}
$$

onde $\underline{X} \sim N_{n}(\underline{1}, \Sigma), \quad \underline{Y} \sim N_{n}(\underline{0}, I)$ $\mathrm{X}=A^{\prime} A$. 
De maneira análoga, $u m$ vetor $X(n \times 1)$ ć clito ter nma distribuição clíptica com parâmetros $\underline{\mu}(n \times 1)$ a $\Sigma(n \times n)$ sc

$$
\underline{X}=\underline{\mu}+A^{\prime} \underline{Y}
$$

onde $\underline{Y}$ tem distribuiçào esférica com média $\underline{0}$ e matriz de dispersão $\mathrm{I}, A: k \times n$ e $A^{\prime} A=\Sigma$ con posto $r(\Sigma)=k$. Denotaremos $\underline{Y} \sim E_{s} s_{n}(\underline{0}, I)$ e $\underline{X} \sim F l_{n}(\underline{\mu}, \Sigma)$. (Ver Fang. Kotz e Ng (1990), Definição 2.2. pg. 31]

\subsection{A distribuição t-student multivariada}

Sejam $\underline{Z} \sim N_{n}(\underline{0}, \Sigma) . \Sigma$ con posto completo, on seja, $v(S)=n \cdot V \sim \nu \cdot \frac{1}{\gamma_{\nu}^{2}}$ independentes. Notemos que $\underline{Z}$ pode ser expresso como $\underline{Z}=A^{\prime} \underline{Z}^{(k)}$, onde $A^{\prime} A=\Sigma, r(A)=k$ e $\underline{Z}^{(k)} \sim$ $N_{k}(\underline{0}, I)$. Segunudo esta notaçăo, a distribuição t-student n-variada scrá definida c as suas propriedades listadas.

Definição 3.2 Um uetor alcatón $X(n \times 1)$ f dito ter distribuiçăo t-student n-variada com

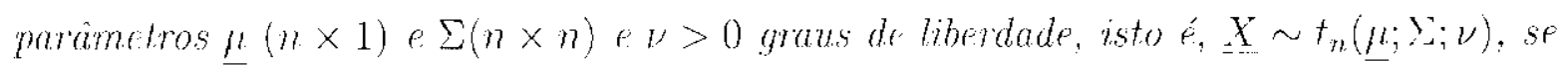

$$
\underline{X}=\underline{\mu}+V \dot{z}
$$

O parametro $\underline{\mu}$ é denominado vetor de locaço a o parametro 2 é denominado matriz de dispersaio.

A função densidade da distribungäo t-student multivariada $t_{n}(u ; \Sigma ; \nu)$ ć dada por:

$$
f(x)=\frac{\Gamma\left[\frac{1}{2}(\nu+n)\right]}{(\pi)^{\frac{n}{2}} \Gamma\left(\frac{1}{2} \nu\right)} \frac{1}{\sqrt{|\Sigma|}} \nu^{\frac{1}{2} \nu}\left\{u+(x-\underline{\mu})^{\prime} \Sigma^{-1}(\underline{x} \quad \underline{\mu}\}^{-\frac{1}{2}(n+\nu)}\right.
$$

Ver. Vallo (1994, j)g. 61).

\subsubsection{Propriedades da t-student}

Considere $\underline{X}$ un vetor aleatório con distribuigào t-student n-variada com vetor de

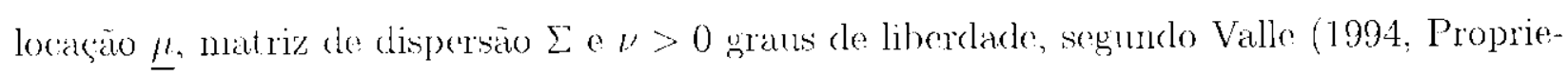
darles 3.2 .2 , pgs. 66 à 72 ) o vetor aleatório $\underline{X}$ verifica as seguintes propriedades: 
(a) $F(\underline{X})$ e $\operatorname{Com}(\underline{X})=E\left(\underline{X X^{\prime}}\right)-E(\underline{X}) E\left(\underline{X}^{-1}\right)$ são dadas por:

$$
E(\underline{X})=\underline{\mu}, \text { se } \quad>>1
$$

e

$$
\operatorname{Cov}(\underline{X})=\frac{\nu}{l-2} \Sigma, \text { se } \nu>2
$$

(b) Deste (1uc $A Z \sim N_{n}\left(\underline{0}, A \Sigma A^{\prime}\right)$ ó independente de $V \sim \nu / x_{1}^{2}$ temos:

$$
\eta+A \underline{X}=\underline{\eta}+A \underline{u}+V^{\prime \frac{1}{2}} A \underline{Z} \sim t_{m}\left(\eta+A \underline{\mu} ; A \Sigma A^{\prime} ; \nu\right)
$$

onde $A$ ce uma matri $\%(m \times n)$ e $\eta \underline{u m}$ vetor $(m \times 1)$. Como $\Sigma$ édefinida positiva, tem-se que

$$
\Sigma^{-\frac{i}{2}}(\underline{X}-\underline{\mu}) \sim t_{n}(\underline{0}: I ; \nu)
$$

Considerando a partição

$$
\underline{X}=\left(\begin{array}{c}
\underline{X}_{1} \\
\underline{X}_{2}
\end{array}\right), \underline{Z}-\left(\begin{array}{c}
\underline{Z}_{1} \\
\underline{Z}_{2}
\end{array}\right), \underline{\mu}=\left(\begin{array}{c}
\underline{\mu}_{1} \\
\underline{\mu}_{2}
\end{array}\right) \quad e \Sigma=\left(\begin{array}{cc}
\Sigma_{11} & \Sigma_{12} \\
\Sigma_{21} & \Sigma_{22}
\end{array}\right)
$$

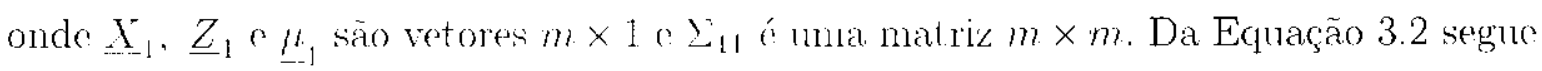
quic

$$
\underline{X}_{1}=\underline{\mu}_{1}+V^{\prime} \underline{Z}_{1} \sim t_{m}\left(\underline{\mu}_{1} ; \Sigma_{11} ; \nu\right)
$$

onde $\underline{Z}_{1} \sim N_{m}\left(\underline{0}, \Sigma_{11}\right)$ é independente de $V \sim \nu \cdot \frac{1}{\chi_{1}^{2}}$. A distribuição marginal de $\underline{X}_{2}$ ́́ obtida analogamente.

(c) Se $\underline{X}$ a particionado como no item anterior, tent-se que a distribuição conclicional de $\underline{X}_{1}$, dado $\underline{X}_{2}=\underline{x}_{2}$ ć t-student m-variada.

(d) $\underline{X} \sim \ln (\underline{\mu} ; \Sigma ; \nu)$ se e somente se para todo $\underline{a}(n \times 1), \underline{a} \underline{X} \sim t_{1}\left(\underline{a} \underline{\underline{a}} \underline{\underline{a}} \underline{a}^{\prime} \Sigma \underline{a} ; \nu\right)$.

\subsection{Distribuições relacionadas}

Aqui, vamos relacionar alguns resultados importantes que serão usados nos próximos capítulos. 
Definição 3.3 Sejam $S_{1} \sim x_{n}^{2} / m \rho S_{2} \sim \nu_{1}^{2}$ independentes. Entäo, $F=S_{1} S_{2} \sim F_{n . \nu}$ e tem densidade dada por:

$$
f(x)=\frac{\Gamma\left[\frac{1}{2}(n+\nu)\right]}{\Gamma\left(\frac{1}{2} n\right) \Gamma\left(\frac{1}{2} \nu\right)}\left(\begin{array}{l}
n \\
\nu
\end{array}\right)^{\frac{1}{2} n} x^{\frac{1}{2}(n-2)}\left[1+\frac{n}{\nu} x\right]^{-\frac{1}{2}(n-\nu)} ; x>0 .
$$

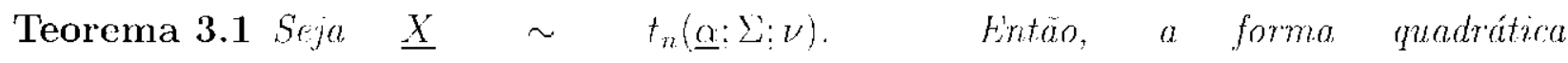
$(\underline{X}-\underline{\alpha})^{\prime}(\Sigma)^{-1}(\underline{X}-\underline{\alpha}) \sim n \cdot F_{n, \nu}$.

Prova: Temos due

$$
\underline{X} \sim t_{n}(\underline{x}: \Sigma: \nu) \Rightarrow \underline{Y}-\underline{a} \sim t_{n}(\underline{0} ; \Sigma ; \nu)
$$

c queremos nucontraj a distribuição de $(\underline{X}-\underline{a})^{\prime}(2)^{-1}(\underline{X}-\underline{\underline{k}})$. Utilizando a Definição 3.2 podemos encererer

$$
\underline{X}-a=\underline{0}-V^{\frac{1}{2}} \underline{Z}
$$

onde $\underline{Z} \sim N_{n}(\underline{0}, \Sigma), \operatorname{com} r(\Sigma)=n \cdot V \sim \nu \cdot \frac{1}{x_{\nu}^{2}}$

Então:

$$
\begin{aligned}
(\underline{X}-\underline{\alpha})^{\prime}\left(\Gamma \Sigma \Gamma^{\prime}\right)^{-1}(\underline{X}-\underline{\alpha}) & =\left(V^{\frac{1}{2}} \underline{Z}\right)^{\prime}(\Sigma)^{-1}\left(V^{\frac{1}{Z}} \underline{Z}\right) \\
& =\underline{Z}^{\prime} V^{\frac{1}{2}}(\Sigma)^{-1} V^{2} \underline{Z} \\
& =V^{\prime} \underline{Z}^{\prime}(\Sigma)^{1} \underline{Z} .
\end{aligned}
$$

Podemos observar que a forma quadrática tem distribuição qui-quadrado, on seja,

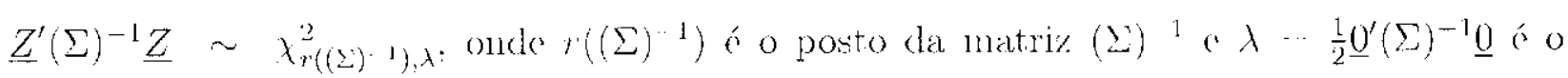

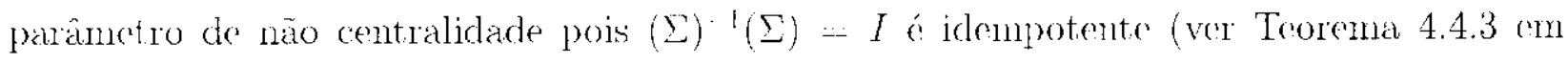
Graybill (1976), pg. 135). Temos por hipótese g110 $\underline{L} \sim N_{n}(\underline{0}, \Sigma)$ a $V \sim v \cdot \frac{1}{\lambda_{\nu}^{2}}$ são independentes.

$$
\log O,(\underline{X}-\underline{\alpha})^{\prime}(\Sigma){ }^{\prime}(\underline{X}-\underline{\alpha}) \sim n \cdot k_{n, \nu}
$$




\section{Capítulo 4}

\section{Modelo Estatístico e Estimação}

Neste capítulo, vamos apresentar $u$ modelo estatístico adequado para explicar os dados e obter o cstinnador de monnentos e de máxina verossinilhança para a temdênecia alas medições de cada laboratório en relaşäo ao valor do referenciat.

\subsection{Modelo proposto}

Considere um programa de comparaçäo interlaboratorial com um grupo de $N$ laboratórios onder cada laboratólio realiza un grupo de mediçöes de um mesmo padrào, tal que:

$$
Y_{i j}=\alpha_{i}+X+\epsilon_{i j} \quad\left\{\begin{array}{l}
j=1, \cdots, n_{i} \\
i=1, \cdots, N,
\end{array}\right.
$$

onde $X$ é uma variável aleatória con módia $\mu_{a}$ e desvio padrāo $\sigma_{2}, \alpha_{i} \in \mathbb{R}$ (? $\epsilon_{i j}$ săo variáveis aleatórias com média 0 e desvio padräo $\sigma_{i}>0$ para todo $j=1, \cdots, n_{i}$ e $i=1, \cdots, N$. O equipannento padrão á representado pela variável alcatória $X$ e as mediçóes do i-ćsimo lábo-

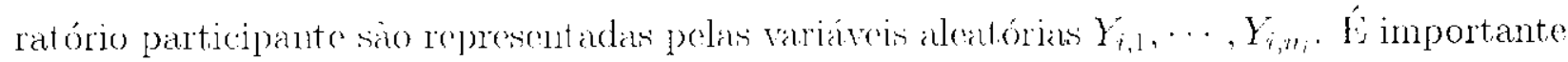

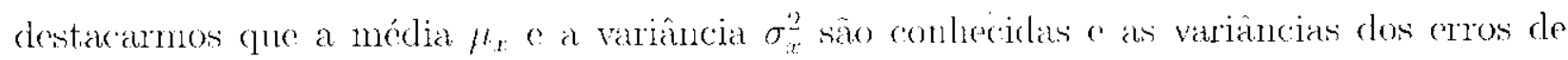
mediçós dos laboratórios $\sigma_{i}^{2}$ também são conllecidats.

Desdo que cada laboratório tem equipamentos de mediçăo, condiçóos ambientais e operadores distintos, e as réplicas, em cada laboratório, são obtidas em condições de repetitividade, vamos assumir, conforme Jaceh (1985, pg. 43), cine:

- $\epsilon_{i j}$ e $\epsilon_{k j}$ sĩo não corrolacionados para todo $i, k=1, \cdots, N$ e j-1, , , , $n_{i}$; 
- $\epsilon_{i j}$ e $\epsilon_{i l}$ são năo correlacionados para todo $i=1, \cdots, N$ e $j, l=1, \cdots, n_{i}$;

- $\epsilon_{i j}$ e $X$ são não correlacionados para todo $i=1, \cdots, N \cap j=1, \cdots, n_{i}$.

Com isso. temos que:

$$
E\left(\epsilon_{i j} \epsilon_{k j}\right)=0 \quad, \quad E\left(\epsilon_{i j} \epsilon_{l l}\right)=0 \quad \text { ○ } E\left(\epsilon_{i j} X\right)=0
$$

para todo $i, k=1, \cdots, N$ i $; l=1, \cdots, n_{i}$.

Além disso, vanos assimmir que

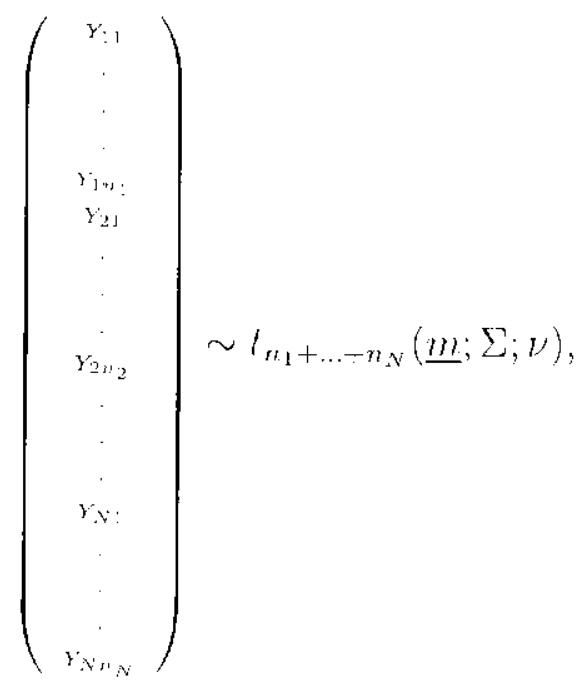

onde $\nu>0$ ć o gram de liberdade. $\Sigma$ a matriz de dispersão e m o vetor de locação.

Açui,

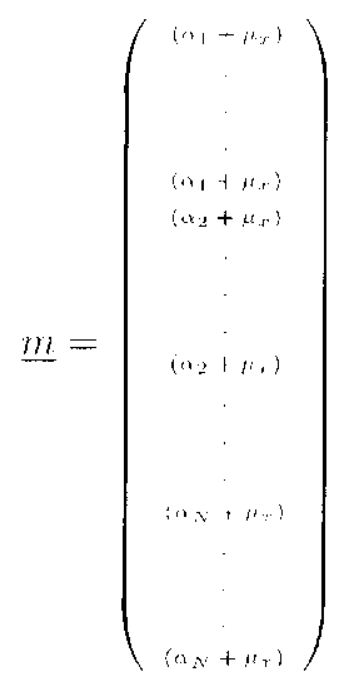

e 


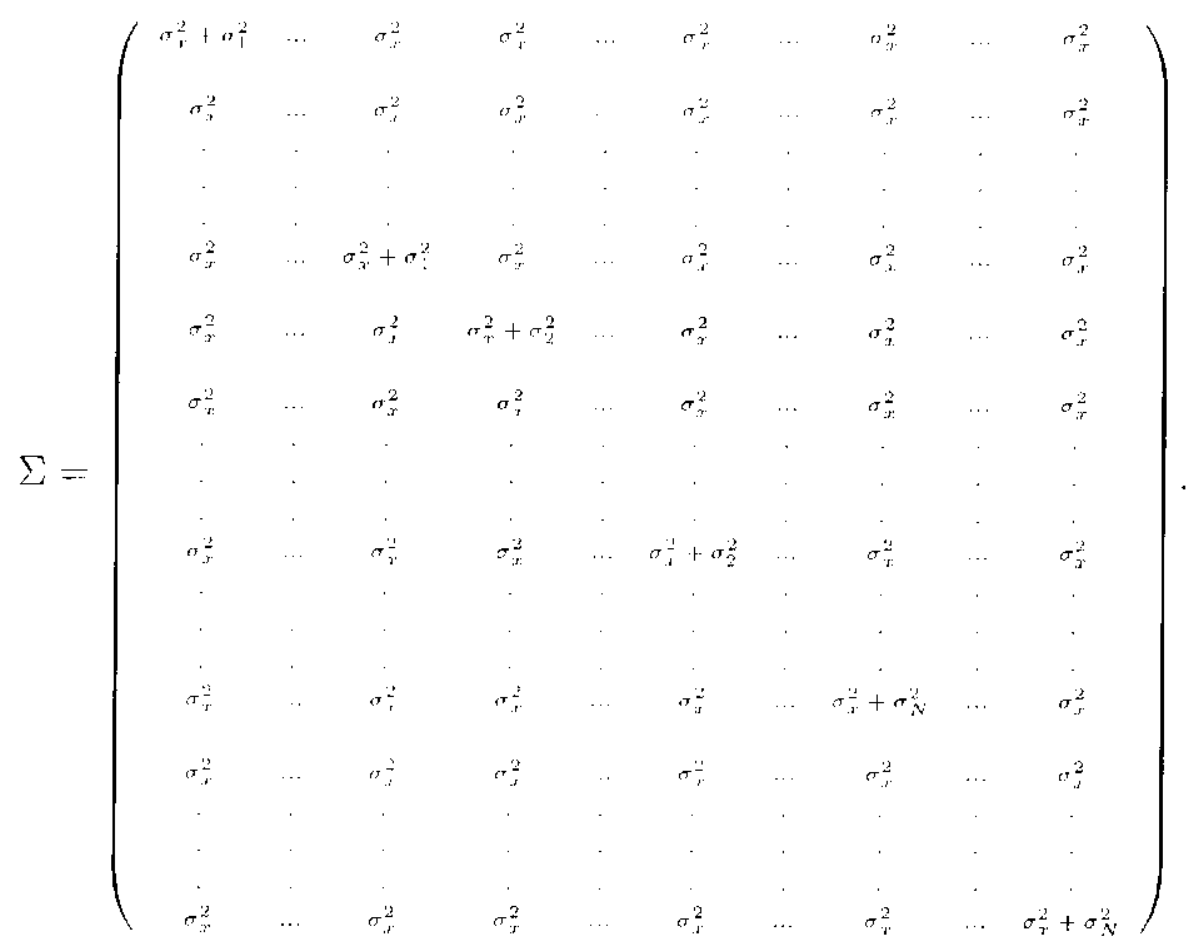

No nosso caso, a matriz de dispersão a contecida, porém, o gran de liberdade e as tendêneias $\alpha_{i}$ de cata laboratório são parânetros desconlecidos. Para simplificar nonsa análisce vamos denotar o vetor de medições por

$$
\underline{Y}=\left(\begin{array}{c}
\underline{Y_{1}} \\
\underline{Y_{2}} \\
\cdot \\
\cdot \\
\cdot \\
\underline{Y}_{N}
\end{array}\right): \text { ondr } Y_{i}=\left(\begin{array}{c}
Y_{i 1} \\
\cdot \\
\cdot \\
\cdot \\
Y_{i n .}
\end{array}\right)
$$

para $i=1, \cdots, N$. Seja $\underline{1_{i}}$ unn vetor $n_{i} \times 1$ com elementos nuitários. Com isso

$$
\underline{\alpha_{i}}=\underline{1_{i}} \alpha_{i}=\left(\begin{array}{c}
\alpha_{i} \\
\cdot \\
\cdot \\
\cdot \\
\alpha_{i}
\end{array}\right) \cdot\left(\begin{array}{c}
\underline{\alpha_{1}} \\
\underline{\alpha_{2}} \\
\cdot \\
\cdot \\
\cdot \\
\underline{\alpha_{N}}
\end{array}\right)=\underline{a}^{*},
$$


onde $i=1, \cdots, N$. Além disso.

$$
\underline{\mu_{x}}(i)=\underline{1}_{i} \mu_{x}=\left(\begin{array}{c}
\mu_{x} \\
\cdot \\
\cdot \\
\mu_{x}
\end{array}\right) \log 0,\left(\begin{array}{c}
\mu_{x}(1) \\
\mu_{x}(2) \\
\cdot \\
\cdot \\
\cdot \\
\mu_{x}(N)
\end{array}\right)=\underline{\mu_{x}^{*}}
$$

onde $i=1, \cdots, N$. A partir disso, temos (unc $\underline{m}=\alpha^{*}+\underline{\mu_{x}^{*}}$.

Portianto, temos que $\underline{Y} \sim t_{n_{1}+\ldots n_{N}}\left(\underline{\alpha}^{*}+\underline{\mu}_{x}{ }^{*}: \Sigma ; \nu\right)$ on seja, $\underline{Y}$ tem distribulção tstudent $L$ variada, onde $L==n_{1}+\ldots+n_{N}$. Além disso, vamos denotar $\Sigma$ como uma matriz particionada. lia forma:

$$
\Sigma=\left(\begin{array}{ccccc}
\Sigma_{11} & \Sigma_{12} & \Sigma_{13} & \ldots & \Sigma_{1 N} \\
\Sigma_{21} & \Sigma_{22} & \Sigma_{233} & \ldots & \Sigma_{2 N} \\
\Sigma_{31} & \Sigma_{32} & \Sigma_{333} & \ldots & \Sigma_{3 N} \\
\cdot & \cdot & \cdot & \ldots & \cdot \\
\cdot & \cdot & \cdot & \ldots & \cdot \\
\cdot & \cdot & \cdot & \ldots & \cdot \\
\Sigma_{N 1} & \Sigma_{N 2} & \Sigma_{N 3} & \ldots & \Sigma_{N N}
\end{array}\right)
$$

tial cule

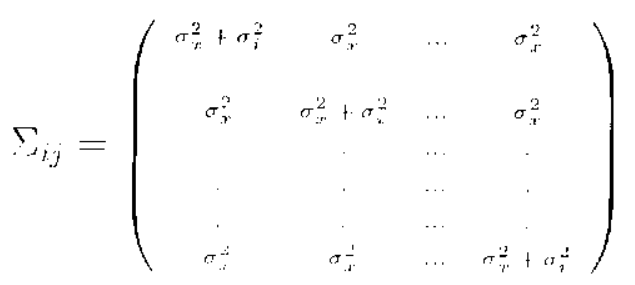

parar $i=j$ com dimenáa $n_{i} \times n_{i}$. Agora, para $i \neq j$, temos que

$$
\sum_{i j}=\left(\begin{array}{cccc}
\sigma_{r}^{2} & \sigma_{i}^{\prime} & \ldots & \sigma_{r}^{2} \\
\sigma_{r}^{2} & \sigma_{r}^{2} & \ldots & \sigma_{r}^{2} \\
& \sigma_{r}^{2} & \ldots & \vdots \\
\sigma_{r}^{2} & \sigma_{r}^{2} & \ldots & \sigma_{r}^{2}
\end{array}\right)
$$

com dimensão $n_{i} \times n_{j}$. Além disso, $\Sigma_{i j}=\Sigma_{j i}$ para $i, j=1, \ldots, N$. 
A firm do encontrarmos a inversa de $\Omega$. verificamos que a mesma pode ser expressia da seguinte maneira

$$
\Sigma=A+\sigma_{r}^{2} \cdot \underline{1_{L}} \cdot \underline{1_{L}{ }^{\prime}}
$$

onde $\underline{1_{L}}$ é $1 \mathrm{~m}$ vetor $L \times 1$ de $111 \mathrm{~s}$, $\sigma_{a}^{2}$ à variância do $X$ e

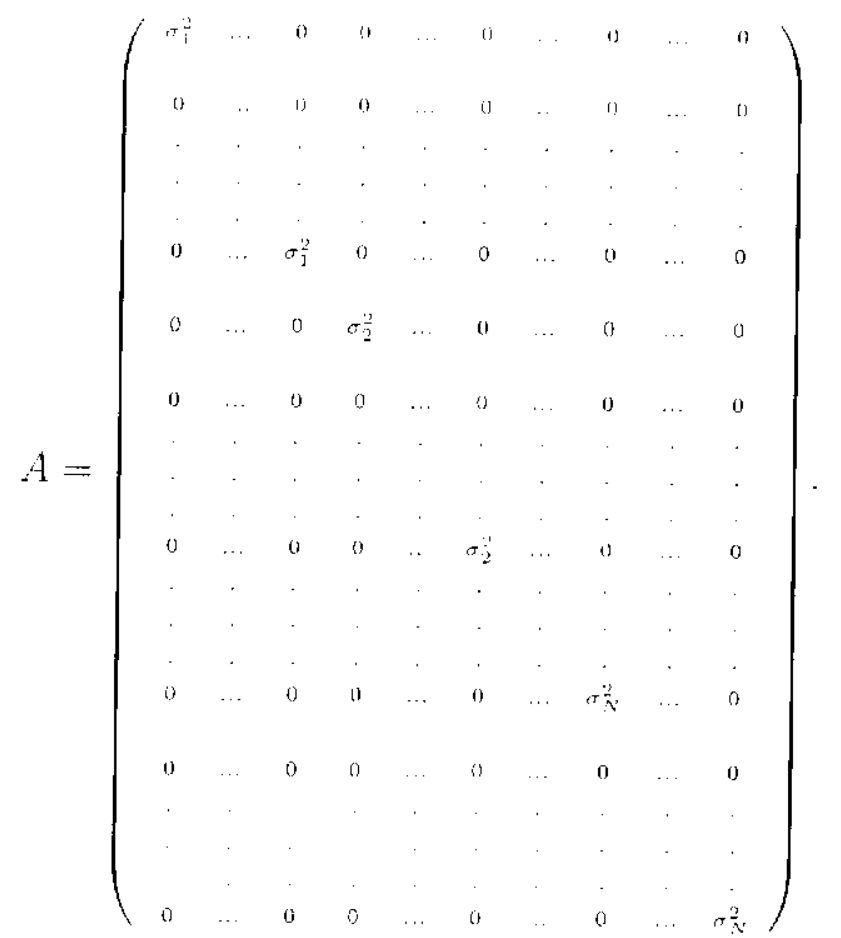

Utilizando resultados para inversas de matrizes, apresentados por Graybill (1983, Teoremla 8.3.3, pg. 189), tenos que:

$$
\Sigma^{\cdots 1}=A^{-1}+\gamma \cdot \underline{b}^{*} \cdot \underline{b}^{* \prime}
$$

onde $A^{-1}$ í a inversa da matriz diagonal $A$.

$$
\gamma=-\sigma_{r}^{2}\left(1+\sigma_{r}^{2} \cdot \sum_{i=1}^{N} \frac{n_{i}}{\sigma_{i}}\right)^{-1} \quad 0 b^{*}=\left(\begin{array}{c}
\frac{b_{1}^{*}}{b_{2}^{*}} \\
\cdot \\
\cdot \\
\cdot \\
\underline{b_{N}^{*}}
\end{array}\right)
$$

$\operatorname{coll} \underline{b_{i}^{*}}=\underline{1_{i}} \frac{1}{\sigma_{i}^{*}}: \operatorname{parad} i=1 \ldots, N$. 
Vamos particionar esta inversa da seguinte maneira:

$$
\Sigma^{-1}=\left(\begin{array}{ccccc}
\Delta_{11} & \Delta_{12} & \Delta_{13} & \ldots & \Delta_{1 N} \\
\Delta_{21} & \Delta_{22} & \Delta_{23} & \ldots & \Delta_{2 N} \\
\Delta_{31} & \Delta_{32} & \Delta_{33} & \ldots & \Delta_{3 N} \\
\cdot & \cdot & \cdot & \ldots & \cdot \\
\cdot & \cdot & \cdot & \ldots & \cdot \\
\cdot & \cdot & \cdot & \ldots & \cdot \\
\Delta_{N 1} & \Delta_{N 2} & \Delta_{N 3} & \ldots & \Delta_{N N}
\end{array}\right),
$$

onde $\Delta_{i i}$ é nma matriz simétrica $n_{i} \times n_{i}$ com elementos da diagonal ignais a

$$
\frac{|\Sigma|-\sigma_{z}^{2}\left(\sigma_{1}^{2}\right)^{n_{i}} \ldots\left(\sigma_{i}^{2}\right)^{n_{1}-1} \ldots\left(\sigma_{N}^{2}\right)^{n_{N}}}{|\Sigma| \sigma_{i}^{2}}
$$

e o restante

$$
\frac{-\sigma_{x}^{2}\left(\sigma_{1}^{2}\right)^{n_{1}} \ldots\left(\sigma_{i}^{2}\right)^{n_{1}-2} \ldots\left(\sigma_{N}^{2}\right)^{n_{N}}}{! 2 i}
$$

para $i=1, \ldots, N$. No caso de $i \neq l$, para $i, l=1, \ldots, N$, temos que $\Delta_{i l}=\Delta_{l i}$, com clementos igntais al

$$
\frac{-\sigma_{x}^{2}\left(\sigma_{1}^{2}\right)^{n}: \ldots\left(\sigma_{i}^{2}\right)^{n_{1}-1} \ldots\left(\sigma_{l}^{2}\right)^{n_{l}-1} \ldots\left(\sigma_{N}^{2}\right)^{n_{N}}}{! \Sigma}
$$

onde

$|\Sigma|=\left(\sigma_{1}^{2}\right)^{n_{1}-1} \ldots\left(\sigma_{N}^{2}\right)^{n_{N}-1}\left(\sigma_{1}^{2} \ldots \sigma_{N}^{2}+\sigma_{3}^{2}\left[n_{1} \sigma_{2}^{2} \sigma_{3}^{2} \ldots \sigma_{N}^{2}+n_{2} \sigma_{1}^{2} \sigma_{3}^{2} \ldots \sigma_{N}^{2}+\ldots+n_{N} \sigma_{1}^{2} \sigma_{2}^{2} \ldots \sigma_{N-1}^{2}\right]\right)$

é o deteminante de $\Sigma$ [ver Trorema 8.4.3 em Graybill (1983), pg. 203].

A scguir vamos estimar os parâmetros de tendência $\left(\alpha_{i}\right)$ en relação ao valor de referência (X) e o parâmetro $\nu$.

\subsection{Estimador para os Parâmetros do Modelo}

De acordo com o modelo proposto nat secia anterior, vanos apresentar os estimadores para o parsinctro de tendénelia $a_{i}$ e para o parânetro 


\subsubsection{Estimador de Momentos}

Estimador de Momentos para o parâmetro de tendência $\alpha_{i}$

Nonso modelo é

$$
Y_{i j}=\alpha_{i}+X+\epsilon_{i j} ; j=1, \ldots n_{i} \text { e } i=1, \ldots, N
$$

onde $X$ uma variável alcatória com média $\mu_{x:}$ " desvio padrão $\sigma_{x}, \alpha_{i} \in \mathbb{R}$ c $\epsilon_{i j}$ são variáveis aleatórias com média 0 e desvio padrão $\sigma_{i}>0$. Sabemos q11e $E\left(Y_{i j}\right)=\alpha_{i}+\mu_{x}$ e vamos definir $Y_{i}=\frac{\sum_{j=1}^{n_{i}} Y_{j}}{n_{i}}$ e $\bar{y}_{i .}=\frac{\sum_{j-1}^{n_{i}} y_{i j}}{n_{i}}$. Ltilizando o método de momentos, temos que

$$
\begin{aligned}
E\left(Y_{i j}\right) & \cdots \\
\alpha_{i}+\mu_{x} & -\frac{\sum_{j=1}^{n_{i}} y_{i j}}{n_{i}} \\
a_{i} & =\frac{\sum_{j=1}^{n_{i}} y_{i j}}{n_{i}}-\mu_{x} \\
\hat{a}_{i} & =\bar{y}_{i}-\mu_{i r} .
\end{aligned}
$$

Portanto, $\hat{\sigma}_{i}=\bar{Y}_{i}-\mu_{r}$ í o cstimador de momentos para $\alpha_{i}$.

\section{Estimador de Momentos para o parâmetro $\nu$}

Utilizando o método de momentos, onde igualamos o segundo momento amostral com o segundo momento populacional, encontramos que o estimador para o parâmetro ly será nulo (ver Apêndice A ). Desta forma, vamos traballiar o estudar as propriedades do estimador de máxina verossimilhança deste parànetro.

\subsubsection{Estimador de Máxima Verossimilhança (EMV) \\ EMV para o parâmetro $\alpha_{i}$}

Para o modelo proposto, a função de verossimilhança é dada por :

$$
\begin{aligned}
& f\left(\underline{y}: \underline{\alpha}^{*}, \nu\right)=\frac{\Gamma\left[\frac{1}{2}(\nu+L) ?\right.}{(\pi)^{\frac{l}{2}} \Gamma\left(\frac{1}{2} \nu\right)}-\frac{1}{\sqrt{\lfloor\mid}} \nu^{\frac{1}{2} \nu}\left\{\nu+(\underline{y} \cdots \underline{m})^{\prime} \Sigma^{-1}(\underline{y}-\underline{m})\right\}^{-\frac{1}{2}(l+\nu)}
\end{aligned}
$$

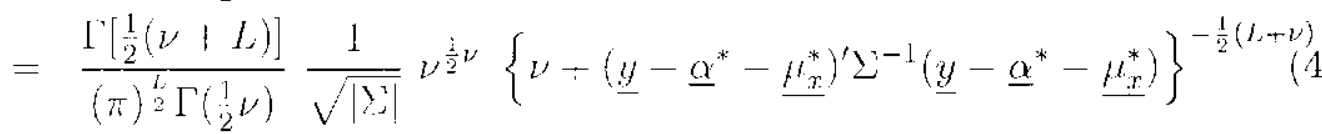


Tomamos $\underline{y}-\underline{\alpha}^{*}-\underline{\mu}_{x}^{*}$ da seguinte forma:

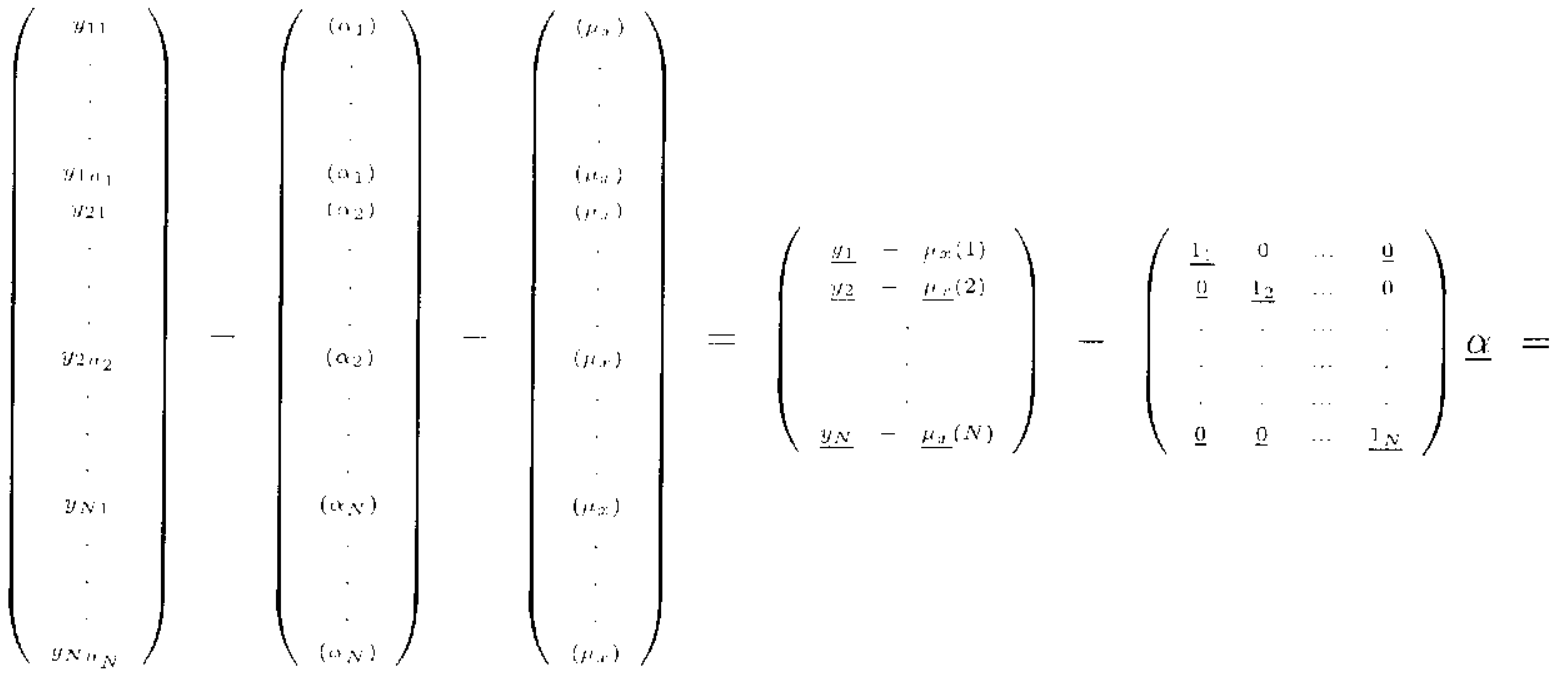

$$
\begin{aligned}
& =\left(\underline{y}-\underline{\mu}^{*}\right)-J \underline{\alpha} .
\end{aligned}
$$

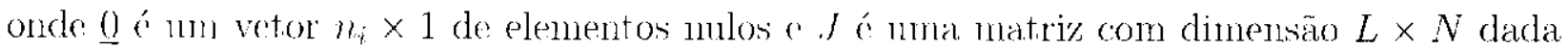
por:

$$
J=\left(\begin{array}{cccc}
\underline{1_{1}} & \underline{0} & \cdots & \underline{0} \\
0 & \underline{1_{2}} & \cdots & \underline{0} \\
\cdot & \cdot & \cdots & \cdot \\
\cdot & \cdot & \cdots & \cdot \\
\cdot & \cdot & \cdots & \cdot \\
\underline{0} & \underline{0} & \cdots & \underline{1_{N}}
\end{array}\right)
$$

Assim. obtemos que $\underline{\alpha}^{*}=J \underline{\alpha}$. Portanto, a verossimilhança é dada por:

$f(\underline{y} ; n, \nu)=\frac{\Gamma\left[\frac{1}{2}(\nu+L)\right]}{(\pi)^{\frac{L}{2}} \Gamma\left(\frac{1}{2} \nu\right)} \frac{1}{\sqrt{|\Sigma|}} \nu^{1}{ }^{\nu}\left\{\nu+\left(\left(\underline{y}-\underline{\mu x}^{*}\right)-J_{\underline{\alpha}}\right)^{\prime} \Sigma^{-1}\left(\left(\underline{y}-\underline{\mu}_{x}^{*}\right)-J \underline{\alpha}\right)\right\}^{-\frac{1}{2}(L+\nu)}$.

Denotamos por $l\left(\underline{y}: \underline{a}, l^{\prime}\right)=\ln f(\underline{y} ; \underline{\underline{\alpha}}, \nu)$ temos

$$
l(\underline{y}: \underline{\underline{x}}, \nu)=\ln R-\frac{1}{2}(L+\nu) \ln \left\{\nu+\left(\underline{y}-\underline{\mu_{x}}\right)^{\prime} \Sigma^{-1}\left(\underline{y}-\underline{\mu}_{x}^{*}\right)-2\left(\underline{y}-\underline{\mu_{x}}\right)^{\prime} \Sigma^{-1} J \underline{\alpha}+(J \underline{\alpha})^{\prime} \Sigma^{-1} J \underline{\underline{\alpha}}\right\}:
$$

onde $R=\frac{\left.I^{\prime} \frac{1}{2}(\nu-L)\right]}{(\pi)^{\frac{L}{2}}-\left(\frac{1}{2} \nu\right)} \frac{1}{\sqrt{\mid \Sigma !}} \nu^{\frac{1}{2} \nu}$

Derivando I $(\underline{y} ; \underline{\underline{\alpha}}, \nu)$ en relaçäo à $\underline{\alpha}$, temos:

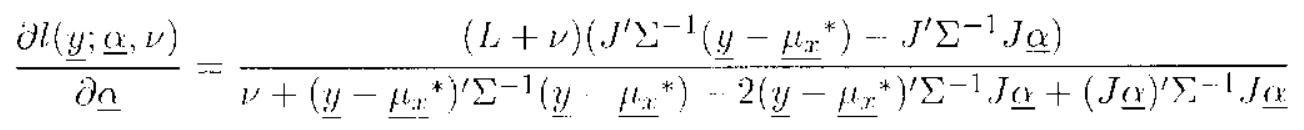


Ao tomarmos $\frac{\partial l(y: \underline{\alpha}, \nu)}{\partial \underline{\underline{q}}, \nu}=0$, obtemos que

$$
\underline{\underline{u}}=\left(J^{\prime} \Sigma^{1} J\right)^{-1} \cdot J^{\prime} \Sigma{ }^{1}\left(\underline{y}-\underline{\mu}_{x}^{*}\right)
$$

Atravós de manipulações algóbricas, temus que

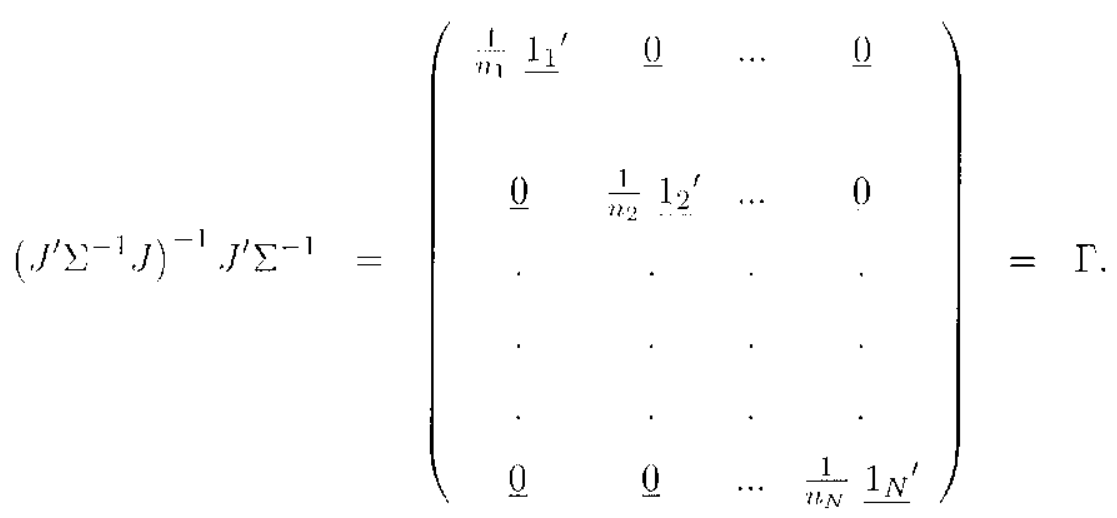

Portanto.

$$
\underline{\hat{\alpha}}=\Gamma\left(\underline{y} \cdots \mu_{r}^{*}\right) .
$$

Con isso, o estimador de máxima verossinilhança é dado por

$$
\underline{\hat{Y}}=\Gamma\left(\underline{Y}-\underline{\mu}^{*}\right)
$$

ou seja,

$$
\left(\begin{array}{c}
\dot{a}_{1} \\
\hat{\alpha}_{2} \\
\cdot \\
\cdot \\
a_{N}
\end{array}\right)=\left(\begin{array}{c}
\bar{y}_{1}-\mu_{r} \\
\bar{Y}_{2 .}-\mu_{x} \\
\cdot \\
\cdot \\
Y_{N}-\mu_{x}
\end{array}\right) .
$$

onde $Y_{i}=\frac{\sum_{j}^{n} \cdot 1 \cdot Y_{i j}}{n_{i}} ; \quad i=1, \cdots, K$. Observe que o Estimador de Máxima Verossimilhança (EMV) para a tendencia das mediçóes coincide com o estimador de momentos. (Observe que o EMV para o parâmetro e năo dependo do grau de liberclade.)

Podemos observar que o estinador é năo viciado. Para o modelo proposto temos que o est inarlor $\therefore$ larto por:

$$
\begin{aligned}
\hat{\alpha}_{i} & =\bar{y}_{i \cdot-} \mu_{x} \\
& =\alpha_{i}+X+\bar{\epsilon}_{i .}-\mu_{x} \\
& =\alpha_{i}+\left(X-\mu_{x}\right)+\epsilon_{i .}
\end{aligned}
$$


onde $\bar{\epsilon}_{i,}=\sum_{\frac{l=1}{n_{i} \epsilon_{i j}}}^{n_{i}}, i=1, \cdots, K$. Logo. $E\left[\hat{\alpha}_{i}\right]=\alpha_{i}$.

Na sequî̀ncia, vamos encontrar a distribuição do EMV do parâmetro de tenclència $\alpha_{i}$. Para isto, utilizamos a propriedade (b) clo Capítulo 3, subseção 3.2

Sabemos que $\underline{Y} \sim t_{L}\left(\underline{\alpha}^{*}+\mu_{x}{ }^{*} ; \Sigma: \nu\right) \log 0, \underline{Y}-\mu_{r}^{*} \sim t_{L}\left(\underline{\alpha}^{*} ; \Sigma ; \nu\right)$ e $\underline{\hat{\alpha}}=\Gamma(\underline{Y}-$ $\left.\underline{\mu}_{x}^{*}\right) \sim t_{N}\left(\Gamma^{\top} \underline{\alpha}^{*} ; \Gamma \Sigma \Gamma^{\prime} ; \nu\right)$.

Portanto,

$$
\underline{\underline{x}} \sim \operatorname{l}\left(\underline{\underline{x}}: \Gamma \Sigma \Gamma^{\prime}: \nu\right)
$$

Scguindo a mesind notaçŭo, vamos agora cucont rar o EXIV para o parâmetro \%.

\section{EMV para o parâmetro $\nu^{\prime}$}

Derivando a cxpressão (4.4) em relação a $\%$, obtemos

$$
\begin{aligned}
\frac{\partial l(\underline{y}: \underline{\alpha}, \nu)}{\partial \nu} & =\frac{\partial\left[\ln \Gamma\left(\frac{1}{2}(\nu+L)\right)\right.}{\partial \nu}-\frac{\partial\left[\ln \Gamma\left(\frac{1}{2} \nu\right)\right]}{\partial \nu}+\frac{1}{2} \ln \nu+\frac{1}{2} \\
& -\frac{1}{2} \ln \left\{\nu+\left(\left(\underline{y}-\underline{\mu_{x}}{ }^{*}\right)-J \underline{\alpha}\right)^{\prime} \Sigma^{-1}\left(\left(\underline{y} \quad \mu_{x}^{*}\right)-J \underline{\alpha}\right)\right\} \\
& +\left(-\frac{1}{2}(L+\nu)\right) \frac{1}{\nu+\left(\left(\underline{y}-\underline{\mu}_{x}^{*}\right)-J \underline{\alpha}\right)^{\prime} \Sigma^{-1}\left(\left(\underline{y}-\underline{\mu}_{\underline{x}}^{*}\right)-J \underline{\alpha}\right)} .
\end{aligned}
$$

Logo.

$$
\begin{aligned}
& \frac{\partial l(\underline{y} ; \underline{\alpha}, \nu)}{\partial \nu^{\prime}}-\frac{\Gamma^{\prime}\left(\frac{1}{2}(\nu+L)\right)}{\Gamma\left(\frac{1}{2}(\nu+L)\right)}-\frac{\Gamma^{\prime}\left(\frac{1}{2} \nu\right)}{\Gamma\left(\frac{1}{2} \nu\right)}+\frac{1}{2}\left\{1+\ln \left[\frac{\nu}{\nu+\left(\left(\underline{y}-\underline{\mu x}_{x}^{*}\right)-J_{\underline{\alpha}}\right)^{\prime} \Sigma^{-1}\left(\left(\underline{y}-\underline{\mu}_{x}^{*}\right)-J \underline{\alpha}\right)}\right]\right. \\
& \left.-\frac{L+v}{\nu+\left(\left(\underline{y}-\mu_{x}^{*}\right)-J \alpha\right)^{\prime} \Sigma^{-1}\left(\left(y-\mu_{x}^{*}\right) \cdots J_{\alpha}\right)}\right\}
\end{aligned}
$$

O estimador $\hat{\nu}$ ć a solução não cxplícita da cquação $\frac{\partial l(\underline{y} ; \underline{\hat{\alpha}}, \hat{y})}{\partial t}=0$. No Capítulo 6, encontraremos uma aproxinıação para a estimativa de máxima verossimilhança para o nosso conjunto de dados, usando unn algoritmo de solução numérica.

\subsection{Consistência dos estimadores de $\alpha_{i}$}

Através da expressão (4.5). temos que

$$
\dot{r}_{i}=\alpha_{i}+\left(X-\mu_{x}\right)+\bar{\epsilon}_{i}
$$

para todo $i \ldots 1, \ldots N$. Utilizando a lei forte dos grandes números (Rao, 1987, pg. 14), concluínos que 


$$
\bar{\epsilon}_{i .} \underset{u_{i}}{\rightarrow} 0 . P-q . c .
$$

Com isso, obtermos que

$$
\alpha_{i} \underset{n_{i} \uparrow \infty}{\rightarrow} \alpha_{i}+\left(X-\mu_{n}\right) . \quad P \quad q . e
$$

Assim. o estinador de máxima verussimilhanģa á năo consistente. Porém ao analisarmos o 'xperimento do ET' o o modelo proposto para descrever os dados, podemos concluir que a não consistrincia ó esperada.

Na práticia, o valor de referência é deterninado por consenso on calibração por um laboratório da RIBC. Porém, o valor de referência é uma variável aleatória, cujos parảnetros são conhecidos, conforme proposto pelo ISO/1EC Guide 43-1 (1997). Como o valor de referencia "varia" durante a realizaçio do programa de EP, mesmo que o número de mediçòes do laboratório tenda ao infinito, nào temos como garantir a consistência do estimador $\hat{x}_{i}$. 


\section{Capítulo 5}

\section{Testes de hipóteses}

Nerste capítulo desenvolveremos testes com o propósito de verificar a consistencia do grupo de laboratórios participantes do programa de FP e a competencia de cada laboratório individualmente.

\subsection{Teste individual para os laboratórios.}

A competència do cada laboratório será testada utilizando as seguintes hipóteses:

$$
\left\{\begin{array}{l}
H_{0}: \alpha_{i}=0 \\
H_{1}: \alpha_{i} \neq 0
\end{array}\right.
$$

Observando o resultado (4.6): verificamos que:

$$
\hat{\underline{\alpha}} \sim t_{N}\left(\underline{\alpha}: \Gamma \Sigma \Gamma^{\prime} ; \nu\right)
$$

Pela distribuição dladla em (3.3) temos que:

$$
\hat{\alpha_{i}} \sim t_{1}\left(\alpha_{i} ;\left(\Gamma \Sigma \mathrm{I}^{\prime}\right)_{i j} ; \nu\right)
$$

onde $\left(\Gamma \Sigma \mathrm{I}^{\prime}\right)_{n}$ representa o elemento da i-ćsima linha e i-ésina coluna da matriz de dispersão $\Gamma \Sigma \Gamma^{\prime}$. $\log 0$,

$$
\frac{\alpha_{i}}{\sqrt{\left(\Gamma \Sigma \Gamma^{y}\right)_{i i}}} \sim t_{1}\left(\frac{\alpha_{i}}{\sqrt{\left(\mathrm{I} 2 \mathrm{I}^{\prime}\right)_{i i}}} ; 1 ; \nu\right)
$$

Vanos testar cada laboratório individluahmente utilizando a equaçăo (5.1) encontrada anteriormente e usar a cstinnativa de náxina verossimillanģa $\dot{y}$ para encontrar mua distribuiçăo aproximada, on seja. 


$$
W_{i}=\frac{\hat{r}_{i}}{\sqrt{\left(\Gamma \Sigma \Gamma^{\prime}\right)_{i i}}} \sim t_{1}\left(\frac{\alpha_{i}}{\sqrt{\left(\Gamma^{\prime} \Sigma \Gamma^{\prime}\right)_{i i}}}: 1 ; \nu\right) \approx t_{1}\left(\frac{\alpha_{i}}{\sqrt{\left(\Gamma \Sigma \Gamma^{\prime}\right)_{i j}}} ; 1 ; \hat{\nu}\right),
$$

onde $\left(\Gamma \Sigma \Gamma^{\prime}\right)_{i i}$ representa o clemento da i-ćsina linha o i-ésima coluna da matriz de dispersão $\Gamma \Sigma \Gamma^{\prime}$.

Sob a hipótese $H_{0}$, temos que:

$$
W_{i}=\frac{\dot{\alpha}_{i}}{\sqrt{\left(\Gamma^{Y} \mathrm{I}^{\prime}\right)_{i i}}} \approx t_{1}(0 ; 1 ; \hat{\nu})
$$

onde $\left(\Gamma \Sigma \Gamma^{\prime}\right)_{\text {ir }}$ representa o elemento da i-ésima linha e i-ésima coluna da matrì de dispersão $\Gamma \Sigma \Gamma^{\prime}$. Neste caso, a Região Crítica será dada por:

$$
R C=\left\{W_{i} \in \mathbb{R}: W_{i}<x_{c_{3}} \text { ou } W_{i}>x_{c_{2}}\right\},
$$

e para um valor fixado $0<\eta<1$, determinamos os números $x_{c_{1}}$ e $x_{c_{2}}$ de modo que

$$
\mathrm{P}\left(W_{i}<x_{c_{1}} \text { ou } W_{i}>x_{c_{2}}\right)=\eta
$$

\subsubsection{Poder do Teste}

$O$ assempenho do um teste a modido pela freqüencia com a qual este execula julgamentos corretos. Esse desempenhó ámedido pela função poder. O poder do testee é diado por:

$$
\pi(\underline{\alpha})=P\left(\operatorname{rejcitar} / H_{0} / \underline{\alpha}\right)
$$

on seja,

$$
\int_{\infty}^{x_{1}} f(x) d x+\int_{x_{02}}^{\infty} f(x) d x
$$

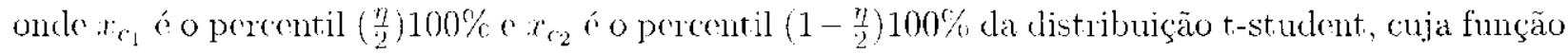
densidade de probabilidade está dada na expressão (6.2) do Capítulo 6.

Fornecendo alguns valores iniciais para $\alpha_{i}$. e usando a expressão (5.2) vamos analisar o desempenho do teste pelo grálico da funçăo poder no Capítulo 6 .

\subsection{Testando o grupo de laboratórios.}

Para avaliar a consistencia do grupo de laboratórios propomos o seguinte teste 


$$
\left\{\begin{array}{l}
H_{0}: \underline{\alpha}=\underline{0} \\
H_{1}: \underline{\alpha} \neq \underline{0} .
\end{array}\right.
$$

Sabmos que $(\underline{\alpha}-\underline{a}) \sim t_{N}\left(\underline{0} ; \Gamma^{\prime} I^{\prime} ; u\right)$. Assim, polo Teorema 3.1 temos que

$$
(\underline{\alpha}-\underline{\alpha})^{\prime}\left(\mathrm{I}^{\prime} \mathrm{I}^{\prime \prime}\right)^{-1}(\underline{\hat{\alpha}}-\underline{\alpha}) \sim N \cdot F_{N, \nu} .
$$

Mais uma vez, tomamos a aproximação

$$
(\underline{\alpha}-\underline{\alpha})^{\prime}\left(\Gamma \Sigma \Gamma^{\prime}\right)^{-1}(\underline{\underline{\alpha}}-\underline{\alpha}) \approx N \cdot F_{N, \hat{\nu}},
$$

onde $\hat{\nu}$ represent a a estimativa de máxima vorossimilhança. Sob $H_{0}$, temos que

$$
W=(\underline{\hat{\alpha}})^{\prime}\left(\Gamma^{2} \mathrm{I}^{\prime}\right)^{1}(\underline{\hat{\alpha}}) \approx N \cdot F_{N, \hat{\nu}}
$$

Neste caso, a Regiāo Crítica será dadła por:

$$
R C=\left\{W \in \mathbb{R}: W>x_{c}\right\}
$$

e para un valor fixado $0<\eta<1$, determinamos o numero $x_{c}$ ale modo que

$$
\mathrm{P}\left(W>x_{c}\right)=\eta
$$

No próximo capítulo, vamos desenvolver estes testes utilizando os dados provenientes do prograna de EP conduriko pola REMLSP na área de eletricidade e avaliar se o grupo de laboratórios f́ consistente or so os labolatórios săo competentes. 


\section{Capítulo 6}

\section{Aplicações}

Neste capitulo, vanos analisar os daclos do prograna de FiP conduzido pela RFMFSP apresentados no Capítulo 2. Como os parâmeros do valor de referencia $\left(\mu_{x}\right.$ e $\left.\sigma_{x}^{2}\right)$ serão obtidos por valor de consenso pela média entre os participantes, vamos detectar a presença de valores extremos, aplicando o teste de Grubbs (1969), para evitar que estes valores extremos vicie nossas estimativas.

O erro normalizado testa as segunintes hipót eses:

$$
\left\{\begin{array}{l}
H_{0}: \quad \text { laboratório compativel } \\
H_{1}: \text { Laboratório não compatível }
\end{array}\right.
$$

on seja, ao fazer o teste indiviclual, se o valor do erro normalizado cair na região de rẹjeç̧ão $\left(E N_{\text {Labi }}>1\right)$, podemos concluir que temos evidenncias para rejeitar $I_{0}$, com nível de confiança de aproximadamente $5 \%$

Proponos ainda as seguintes hipóteses para avaliar a performance individual de cada laboratório con respeito au valor de referéncia,

$$
\begin{cases}H_{0} & : \quad \alpha_{i}=0 \\ H_{1} & : \quad \alpha_{i} \neq 0\end{cases}
$$

para $i=1, \ldots, N$. Se näo rejeitamos $I_{0}$, temos evielencias para dizer que o laboratório está consistente com relação ao valor de referência.

Finalnenter avaliarmos a consisténcia do grupo de laboratórios, testando ats seguintes hipóteses.

$$
\begin{cases}H_{0}: & \alpha_{1}=\alpha_{2}=\ldots=\alpha_{N}=0 \\ H_{1} & : \text { prlo menos um difcrente. }\end{cases}
$$

Se rejeitarmos $H_{0}$, temos evidencias para dizer que o grupo de laboratórios näo á consistente. 


\subsection{Teste de Grubbs}

Como vannos estimar os parânetros do valor de referencia, $\mu_{x}$ e $\sigma_{x}^{2}$, utilizando o procedimento de valor de consenso, a presenga de valores extremos pode viciar nossas estimativas. Para dotectar a presença de valores extremos, vamos aplicar o testo de Grubbs (1969).

Considere $\bar{y}_{1}, \ldots, \bar{y}_{N}$ as médias das mediçóes dos laboratórios participantes. Para detectar a presença de valores extremos, Grubbs (1969) propõe a seguinte estatística de teste

$$
T_{i}=\frac{\left|\bar{y}_{i}-\bar{y}_{. .}\right|}{s} \quad i=1, \ldots, N
$$

onde $\bar{y}_{.}=\frac{\sum_{i=1}^{N} y_{i}}{N}$ "s o desvio padrăo amostral das médias $\ddot{y}_{1}, \ddot{y}_{2}, \ldots, \bar{y}_{N}$. Vamos testar as segrinites liipótcses

$$
\left\{\begin{array}{l}
H_{0}: \text { A observação } i \text { não é um valor extremo } \\
H_{1}: \text { A obscrvação } i \text { é un valor extremo. }
\end{array}\right.
$$

Se $T_{i}>t_{i}$, temos evidencia para rejeitar $H_{0}$ onde $l_{i}$ é un valor que depente da quantidade de observaçôs na anostra, e se cucontra tabelado em Grubbs (1969), com núvel de confiança de 5\% c 1\%. Para os dados do prograna da área de eletricidade, nsimos o nível de confiança de $5 \%$ o

\begin{tabular}{|c|c|c|c|c|c|c|}
\hline Laboratórios & Observaçōes & Mćdia & $\therefore$ & $T$ & 1 & Situaçāo \\
\hline 1 & 1.000008 & 0,999934 & 0,000014699 & $1 .(01193$ & 2,13 & năo ć valor (xtremo \\
\hline 2 & 0.99991 & $0.9999: 34$ & $0.00(01469$ & 0,16612 & 2,13 & năo é valor extremo \\
\hline 3 & 0,99960 & 0,999934 & $0.00(0) 1469$ & 2.27707 & 2,13 & $\therefore$ valor extrono \\
\hline 1 & 0.99998 & 0,999931 & $(0,000) 1469$ & 0,31055 & 2,13 & nádo verlor extreno \\
\hline 5 & 3,000000 & 0.999931 & $(0,0)(0) 1469$ & 0.44674 & 2,13 & nầ ć valor extreno \\
\hline 6 & 0.99990 & 0,999934 & $(0.0001469$ & 0,23421 & 2,13 & näu ć valor extromo \\
\hline 7 & 1,000000 & 0.999931 & 0,0001460 & 0.46145 & 2,13 & não ć valor extromo \\
\hline 8 & $1.00(0)(0)$ & 0.9999931 & $(1.06)(0) 1169)$ & 0.4467 .1 & 2,13 & nào a valor cxliremo \\
\hline
\end{tabular}
rncontramos

Tabela 6.1: Teste de Grubbs

Pela Tabela 6.1 verificamos que a tensão DC estimada pclo laboratório 3 foi considerado valor extremo. com isso, será excluído da nossa análise. 


\subsection{Análise da compatibilidade dos laboratórios}

O valor de consenso pela Média ó um dos proendimentos mais commus an programas de acreditação com EP de rotina. A experiencia do colégio Americano do Patologia concluiu que a média de consenso dos laboratórios participantes é consistente com relação ao valor de referência, e é nuais confiável do que o consenso de laboratórios de referência, ver Tholen (1993). Os parâmetros do valor de referência são determinados conforme abaixo

$$
\mu_{T}=\bar{y}_{.}=\frac{\sum_{i=1}^{N} \overline{y_{i}}}{N} \quad \text { e } \quad \sigma_{r}^{2}=\frac{\sum_{i-1}^{N} \sigma_{i}^{2}}{N} .
$$

Portanto. hy é determinado pela média das médias encontradas pelos laboratórios, o $\sigma_{x}^{2}$ será determinado pela média do quadrado da incerteza combinarla associada às medições dos laboratórios (variância agrupada). Para os nossos dados encontramos os seguintes resultados:

$$
\mu_{x}=0,999982 \quad \text { e } \quad \sigma_{x}^{2}=0,0000001
$$

O erro normalizado, como visto anteriormenté ć dado por:

$$
E N_{\mathrm{Labi}}=\frac{\left|\bar{y}_{i,}-\mu_{x}\right|}{\sqrt{U_{\text {labi }}^{2}+U_{r e f}^{2}}}=\frac{\left|\hat{x}_{i}\right|}{\sqrt{U_{\text {tabi }}^{2}+U_{r e f}^{2}}} \quad i=1, \ldots, K
$$

onde $U_{\text {tubi }}$ o $U_{\text {ref }}$ correspondem às incertezas rexpandidas do laboratório i e do valor de referencia, respectivanente. A incerteza expandicla para o valor de referencia foi estimada como descrito na seção 2.2.4,

$$
U_{r e f}=2 \cdot \sigma_{r}=0.000632
$$

O vetor $\hat{\alpha}$ é dado por:

$$
\hat{\alpha}=\left(\begin{array}{c}
0,0001008 \\
-0,0000722 \\
-0,0000022 \\
0,0000178 \\
-0,0000822 \\
0,0000200 \\
0,0000178
\end{array}\right) .
$$

Os rálenlos dos eros nomalizados e as conchusós en relação a performance de cada laboratório estào descritos na tabela 6.2 no final cleste capítulo. 
Outra forma de testarmos a pertornance individual dos laboratórios á usando a seguinte estatistica:

$$
W i=\frac{\alpha_{i}}{\sqrt{\left(\Gamma \Sigma \Gamma^{\prime}\right)_{i i}}} \approx 1,(0 ; 1 ; i)
$$

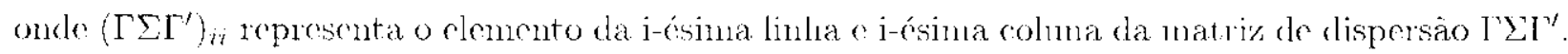
Para os dados provenientes da REMESP, tomos que a matriz de dispersão é dada por:

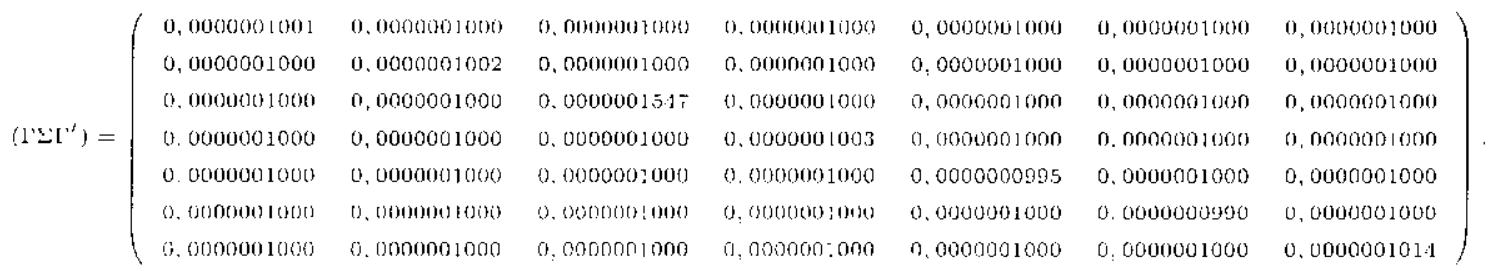

A estimativa de máxima verosisinillança $y$ a a soluçăo não explícita da equação $\frac{\partial l(\underline{y} ; \underline{\underline{\alpha}, \nu})}{\partial \nu}=0$ conforme apresentado no Capítulo 4. subseçāo 4.2.2. Aplicando o algorítmo de Newton-Raphson obtennos $\hat{\nu}=2,72478$. Os cálenlos das estatísticas $W$; estão descritos lia tabela 6.2 no final deste capítulo.

Por último vamos testar a consistência do grupo de laboratórios usando a seguinte estiatística:

$$
W=\underline{\hat{\alpha}}^{\prime}\left(\mathrm{I}^{\prime} \mathrm{L} \mathrm{I}^{\prime}\right)^{-1} \underline{\hat{\alpha}} \approx N \cdot F_{N, \hat{\nu}}^{\prime} .
$$

\begin{tabular}{|c|c|c|c|c|c|}
\hline Teste & $\dot{r}_{i}$ & Fistatistica & $\begin{array}{l}\text { Regiäo } \\
\text { Crítical }\end{array}$ & $U_{l a b i}$ & $E N_{\text {Lalji }}$ \\
\hline Todlos $\times$ Ref. & & 9.809634 & $W>10,3725$ & & \\
\hline Lab $1 \times$ Ref. & 0,0001008 & 0,318707 & $w_{i}<-3,3723$ on $W_{i}>3,3723$ & 0,000020 & 0,159414 \\
\hline Lab $2 \times$ Ref. & $-0,0000722$ & 0,227980 & $W_{i}<-3,3723$ on $W_{i}>3,3723$ & 0,000074 & 0,113926 \\
\hline Lab $4 \times$ Ref. & $-0,0000022$ & 0,005506 & $W_{i}<-3,3723$ on $W_{i}>3,3723$ & 0,001480 & $0,(0) 01367$ \\
\hline Lab $5 \times$ liof. & 0.00000178 & 0,056313 & $W_{i}<-3,3723$ ou $W_{i}>3,3723$ & 0,000082 & 0,027878 \\
\hline Lab $6 \times \operatorname{Ref}$ & $-0,0000822$ & 0.260483 & $\mathfrak{W}_{i}<3,3723$ ou $W_{i}>3,3723$ & 0,000016 & 0,130022 \\
\hline Lab $7 \times$ Ref. & 0.0000200 & $0.06: 3546$ & $w_{i}<-3,3723$ ou $W_{i}>3,3723$ & 0,000058 & 0,031513 \\
\hline Lab $8 \times$ Ref. & 0.0000178 & 0,056006 & $W_{i}<-3,3723$ ou $W_{i}>3,3723$ & 0,000240 & 0,026330 \\
\hline
\end{tabular}

Na próxima tabela, apresentaremos os resultados dos testes individuais o clo conjunto de laboratórios o os erros normalizados.

Tabela 6.2: Teste para avaliar a competencia dos 7 laboratórios 
Na tabela 6.2, observando os erros normalizados a as estatísticas individuais dos laboratórios. temos evidéncias para conchuir que cless vão competentes. Fm relação ao grupo de laboratórios, temos evidências para conchuir que o grupo é consistente com relação ao valor de referéncia.

Apresentamos na seqüiencia o gráfico da funçäo poder para os testes relativos aos laboratórios 1 e 6 . Observando a função densidade da distribuição t-st udent multivariada (3.1). notamos que a fimçăo densidade da distribunçào t-student $t_{1}\left(\frac{\kappa_{\mathrm{z}}}{\sqrt{\left(\Gamma \Sigma \Gamma^{\prime}\right)_{i}}} ; 1 ; \hat{v}\right)$ é dada por:

$$
f(x)=\frac{\Gamma^{2}\left[\frac{1}{2}(\hat{\nu}+1)\right]}{(\pi)^{\frac{1}{2}} \Gamma\left(\frac{1}{2} \hat{\nu}\right)} \hat{\nu}^{\frac{1}{2}} \hat{\nu}\left\{\nu+\left(x-\frac{\alpha_{i}}{\sqrt{\left(\mathrm{I}^{2} \mathrm{~L} \mathrm{I}^{\prime}\right)_{i i}}}\right)\right\}^{-\frac{1}{2}(1+\hat{\nu})} .
$$

Formecemos alguns valores iniciais para $a_{i}$ (eixo das abocissas) o substituminos estes valores na densidade. Aplicamos a função poder dada cun (5.2) a "Inconliamos os seguintes valores para o Poder do Teste do Laboratório 1 (eixo dasis ordemalabs).

\begin{tabular}{|c|c|c|}
\hline$\alpha_{i}$ & $\frac{a_{i}}{\sqrt{\left(\mathrm{l}^{2} \mathrm{I}^{\prime}\right)_{y i}}}$ & $\int_{-\infty}^{-3,3723} f(x) d x+\int_{3,3723}^{\infty} f(x) d x$ \\
\hline-0.00250 & $-7,90,5691$ & 0.988836 \\
\hline-0.00200 & $-6,324555$ & 0,967974 \\
\hline$-0,00150$ & $-4,743416$ & 0,866590 \\
\hline$-0,00100$ & $-3,162278$ & $(0,429045$ \\
\hline$-0,00050$ & 1,581139 & 0,1000070 \\
\hline 0 & () & $(0,049998$ \\
\hline $0.000 \overline{0} 0$ & 1.581139 & 0.100070 \\
\hline 0.00100 & 3,162278 & 0,429045 \\
\hline 0.00150 & 4,743416 & 0,866590 \\
\hline 0.00200 & 6,324555 & 0,967974 \\
\hline 0.00250 & 7,905694 & 0,988836 \\
\hline
\end{tabular}

Tabela 6.3: Cálculo da Função poder para o Lab 1 


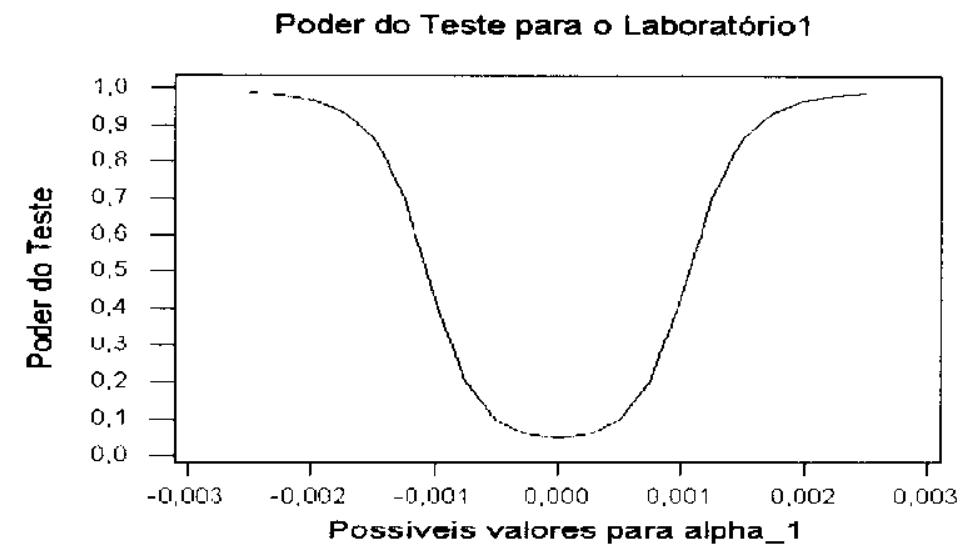

Figura 6.1: Gráfico da Funçăo Poder para o Lab 1

De maneira análoga, calculamos o Poder do Teste do Laboratório 6.

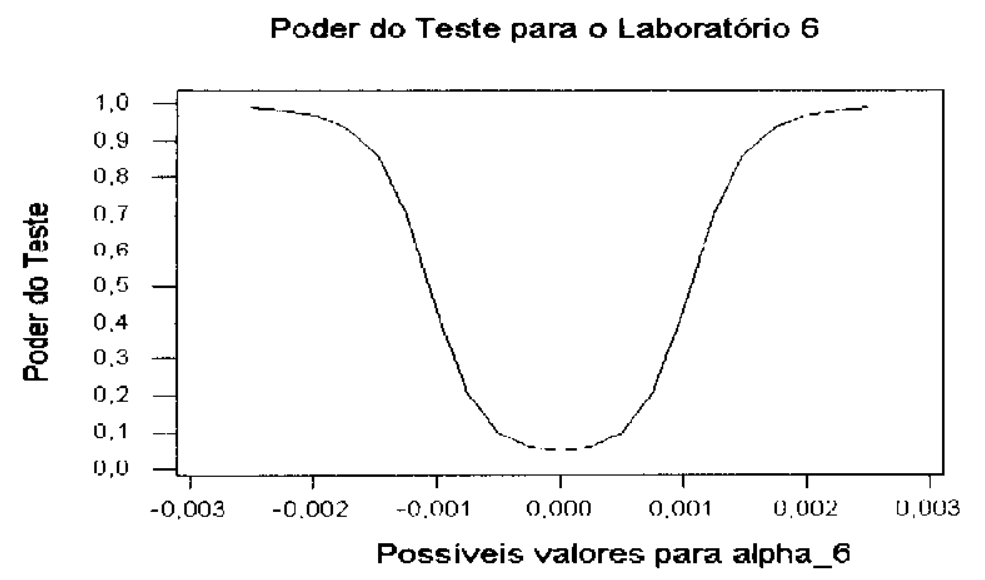

Figura 6.2: Ciráfico da Função Poder para o Lab 6

Observando os gráfico da função poder, concluímos que o teste ć sensivel para uma pequena variaça nos valures de $\alpha_{i}$. 


\section{Capítulo 7}

\section{Considerações finais}

\subsection{Conclusão}

() objetivo do programa de EP é qualificar on nào os laboratórios para realizarem as mediçón descjadas. Analisanos os dados obticlos pela REMESP (Rede de Metrologia de Sào Panlo) na área de eletricidade, cujo mensurado ća tensão DC medida por unn multímetro digital. Neste sentido, 1estamos a consistência do grupo de laboratórios participantes e de cada laboratório individualmente.

Como em medicoes a presonga de valores extremos é constante, modelamos os dados ntilizando a distribuição t-student, para acomodar tais valores extremos. Consideramos aindat un modelo com erro de medigào, variável explicativa aleatória e diferente de outros modelos, estannos interessados em analisar a tendència dos laboratórios participantes do programa ent relação a um valor de referencicia.

() valor de referencia pode ser obtido de diversas naneiras, neste trabalho apresentamos o valor cle refercucia por consenso entre os participantes e neste sentido, foi preciso uma anćlise de valores extrenos. Usamos o método de Grubbs (1969) para identificar valoress cxtremos e somente os laboratórios em que as nediçoes näo foran consideradas como valor extremo continuaram enn nossa análise.

Veste modelo. cstávamos interessados em estimar o gran de liberdade o o parâmetro de tendência da nedição $\alpha_{i}$ do laboratório con respeito ao valor de referência, já que os laboratórios utilizaran sistemas de mediçoes sinnilares para medir o mesmo ítem, com incer- 
teyas determirndas por um processo de calibragäo. Encontramos os estimadores da máxima verossimilhança e de momentos para estes dois parâmetros

Em geral, os resultados dos EP são audlisados via a cstatíatica $E N_{\text {tabibi }}$, descrito na cxpressão (1.1) no Capítulo 1. Desenvolvemos oul ras cstatísticas a avaliamos a compatibilidade entre os laboratórios e a competencia de cada laboratório individualmente. Verificamos a eficácia do treste individual pelo gráfico da funçăo poder. Os resultados estão resumidos na Tabela 6.2.

\subsection{Propostas futuras}

- Gencralizar o modelo para a distribuição elíptica;

- Avaliar a consistència do estimador de máxinna verossinilharcça para o paràmetro y;

- Determinar a distribuiçáo assintótica do estinador de máxima verossimilhança para o parâmetro $\nu$. 


\section{Apêndice A}

\section{Estimador de Momentos para o}

\section{parâmetro $\nu$}

Considere o modelo como anteriormente. Sabemos cun $E\left(Y_{i j}\right)=\alpha_{i}+\mu_{x}$ ' vannos definir

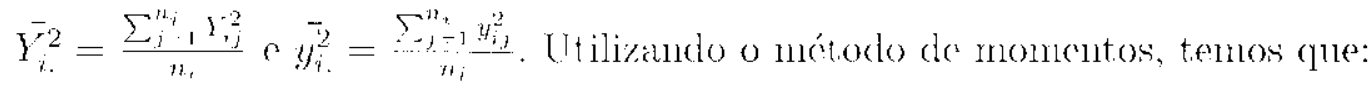

$$
E\left(Y_{i j}^{2}\right)=\overline{y_{i}^{2}}
$$

Sabemos que:

$$
\begin{aligned}
E\left(Y_{i j}^{2}\right) & =\operatorname{Var}\left(Y_{i j}\right)+E^{2}\left(Y_{i j}\right) \\
& =\frac{\nu}{\nu-2}\left(\sigma_{x}^{2}+\sigma_{i}^{2}\right)+\left(\alpha_{i}+\mu_{r}\right)^{2}
\end{aligned}
$$

Enitià,

$$
\begin{aligned}
\frac{\nu}{\nu-2}\left(\sigma_{x}^{2}+\sigma_{i}^{2}\right)+\left(\alpha_{i}+\mu_{x}\right)^{2} & =y_{i}^{2} \\
\sum_{i=1}^{N}\left[\frac{\nu}{\nu-2}\left(\sigma_{x}^{2}+\sigma_{i}^{2}\right)+\left(\alpha_{i}+\mu_{x}\right)^{2}\right] & =\sum_{i=1}^{N} \overline{y_{i}^{2}} \\
\frac{\nu}{\nu-2}\left[\sum_{i=1}^{N}\left(\sigma_{x}^{2}+\sigma_{i}^{2}\right)\right]+\sum_{i=1}^{N}\left(\alpha_{i}+\mu_{x}\right)^{2} & =\sum_{i=1}^{N} \overline{y_{i}^{2}}
\end{aligned}
$$

Logo, 


$$
\begin{aligned}
\nu, \nu & {\left[\sum_{i=1}^{N}\left(\sigma_{x}^{2}+\sigma_{i}^{2}\right)\right]=\sum_{i=1}^{N} \overline{y_{i}^{2}}-\sum_{i=1}^{N}\left(\alpha_{i}+\mu_{x}\right)^{2} } \\
\nu\left[\sum_{i=1}^{N}\left(\sigma_{x}^{2}+\sigma_{i}^{2}\right)\right] & =(\nu-2)\left[\sum_{i=1}^{N} \overline{y_{i}^{2}} \sum_{i=1}^{N}\left(\alpha_{i}+\mu_{x}\right)^{2}\right] \\
\nu\left[\sum_{i=1}^{N}\left(\sigma_{x}^{2}+\sigma_{i}^{2}\right)\right] & =\nu\left[\sum_{i=1}^{N} y_{i}^{2}-\sum_{i=1}^{N}\left(\alpha_{i}+\mu_{x}\right)^{2}\right]-2\left[\sum_{i=1}^{N} \overline{y_{i}^{2}}-\sum_{i=1}^{N}\left(\alpha_{i}+\mu_{x}\right)\right] .
\end{aligned}
$$

Portanto,

$$
\begin{aligned}
\nu & =\frac{-2\left[\sum_{i=1}^{N} Y_{i}^{2}-\sum_{i=1}^{N}\left(\hat{\alpha}_{i}+\mu_{x}\right)^{2}\right]}{\left[\sum_{i-1}^{N}\left(\sigma_{x}^{2}+\sigma_{i}^{2}\right)-\sum_{i-1}^{N} \bar{Y}_{i}^{2}+\sum_{i=1}^{N}\left(\hat{\alpha}_{i}+\mu_{x}\right)^{2}\right]} \\
& =\frac{-2\left[\sum_{i-1}^{N} \bar{Y}_{i}^{2}-\sum_{i=1}^{N}\left(\overline{Y_{i}}-\mu_{x}+\mu_{x}\right)^{2}\right]}{\left[\sum_{i=1}^{N}\left(\sigma_{x}^{2}+\sigma_{i}^{2}\right)-\sum_{i=1}^{N} \bar{Y}_{i}^{2}+\sum_{i=1}^{N}\left(\overline{Y_{i}}-\mu_{x}+\mu_{x}\right)^{2}\right]} \\
& =\frac{-2\left[\sum_{i-1}^{N} \bar{Y}_{i}^{2}-\sum_{i=1}^{N}\left(\overline{Y_{i}}\right)^{2}\right]}{\left[\sum_{i=1}^{N}\left(\sigma_{x}^{2}+\sigma_{i}^{2}\right)-\sum_{i-1}^{N} \overline{Y_{i}}+\sum_{i=1}^{N}\left(\overline{Y_{i}}\right)^{2}\right]}=0 .
\end{aligned}
$$

éo estimador do monemestos para $\nu$. 


\section{Referências Bibliográficas}

[1] ISO/IEC Guide 43-1 (1997) - Proficiency testing by interlaboratory comparisons - Part I: Development and operation of proficiency testing schemes.

[2] ISO GUM (1998) - Guia para expressão da inccrteza em medição, $2^{a}$ ediçăo brasileira.

[3] Fang. K.-T. \& Kot\% S. \& Ng. K. W. Symetrie Multivariate and Related Distributions, Chapman and Hall, 1990.

[4] Graybill, F. A.. Theory and Application on the Linear Model, Duxbury Press, 1976.

[5] Graybill, F. A., Matrices With Applications in Statistics, 2nd edition, 1983.

[6] Grubbs, F. E. (1969) - Procedures for Detecting Ontlying Observations in Samples: Technometrics, 11(1), 1-21.

[7] Jaceh, J. L., Stat istical analysis of measurement curos, Exxon Monograph, John Wiley \& Solls, 1985.

(8) Leão, D. P. J. \& Aoki, R. \& Silva, G. F., Statistical Analysis of Proficiency Testing Results. Notas de estatística do IC.MC - LSP' 2002.

[9] Rao, B. L. S. P.. Asynuptotic Theory of Statistical Inference, , John Wiley \& sons, 1987.

[10] Taylor, J. M. G. \& Little, R. ,J. A. \& Lange, K. L. (1989) - Robust Statistical Modeling (sing the t Distribution, Jonrnal of the Anerican Statistical Association, 84(408). $881-896$

[11] Tholen, D. W. (1993) - Roference values and Panticipant means as Targets in Proficiency lesting. Archives of Pathology and Ialboratory Medicine, 117, 885-889. 
[12] Valle, R. B. A., Distribuiçón Elípticas: Propriedades, Inferência e Aplicações a Modelos de Regressão, Tesce. IME-TSP, 1994. 